\title{
Concepts and practices used to develop functional PLGA-based nanoparticulate systems
}

This article was published in the following Dove Press journal:

International Journal of Nanomedicine

20 February 2013

Number of times this article has been viewed

\section{Hongkee Sah ${ }^{1,2}$ \\ Laura A Thoma ${ }^{2}$ \\ Hari R Desu ${ }^{2}$ \\ Edel Sah ${ }^{3}$ \\ George C Wood ${ }^{2}$}

'College of Pharmacy, Ewha Womans University, Sedaemun-gu, Seoul, South Korea; ${ }^{2}$ College of Pharmacy, The University of Tennessee Health Science Center, Memphis, TN, USA; ${ }^{3}$ College of Science, University of Notre Dame, Notre Dame, IN, USA
Correspondence: Hongkee Sah College of Pharmacy, Ewha Womans University, 52 Ewhayeodae-gil, Sedaemun-gu, Seoul I20-750,

South Korea

Tel +82 232774367

Fax +82 23277285

Email hsah@ewha.ac.kr
Abstract: The functionality of bare polylactide-co-glycolide (PLGA) nanoparticles is limited to drug depot or drug solubilization in their hard cores. They have inherent weaknesses as a drug-delivery system. For instance, when administered intravenously, the nanoparticles undergo rapid clearance from systemic circulation before reaching the site of action. Furthermore, plain PLGA nanoparticles cannot distinguish between different cell types. Recent research shows that surface functionalization of nanoparticles and development of new nanoparticulate dosage forms help overcome these delivery challenges and improve in vivo performance. Immense research efforts have propelled the development of diverse functional PLGA-based nanoparticulate delivery systems. Representative examples include PEGylated micelles/nanoparticles (PEG, polyethylene glycol), polyplexes, polymersomes, core-shelltype lipid-PLGA hybrids, cell-PLGA hybrids, receptor-specific ligand-PLGA conjugates, and theranostics. Each PLGA-based nanoparticulate dosage form has specific features that distinguish it from other nanoparticulate systems. This review focuses on fundamental concepts and practices that are used in the development of various functional nanoparticulate dosage forms. We describe how the attributes of these functional nanoparticulate forms might contribute to achievement of desired therapeutic effects that are not attainable using conventional therapies. Functional PLGA-based nanoparticulate systems are expected to deliver chemotherapeutic, diagnostic, and imaging agents in a highly selective and effective manner.

Keywords: nanoparticulate dosage forms, nanoparticles, polylactide-co-glycolide, functionality

\section{Introduction}

Biodegradable polylactide-co-glycolide (PLGA) nanoparticles have been used as carriers for drugs, peptides, proteins, vaccines, and nucleotides. ${ }^{1}$ Nanoparticles can protect drug moieties from degradation and provide sustained drug release. Nanoparticles are sometimes effective in facilitating intracellular delivery of bioactive materials. Parenteral PLGA nanoparticles have been used for various applications in vaccination, cancer therapy, and the treatment of cerebral disorders. ${ }^{2}$ In particular, nanoparticles have been widely investigated for use in cancer therapy. Nanoparticles, if given intravenously, can extravasate into and accumulate within tumor tissues that have defective blood vessels and impaired lymphatic drainage. ${ }^{3}$ This enhanced permeability and retention (EPR) effect helps direct nanoparticles to tumor sites. ${ }^{4}$ Even though PLGA nanoparticles have emerged as a promising carrier, they have a number of intrinsic drawbacks including the following: 
- Prolonged systemic circulation of nanoparticles is a key prerequisite for maximizing the EPR effect. However, if administered intravenously, bare PLGA nanoparticles are rapidly removed from circulation in the blood, due to opsonization.

- There is increasing demand for the development of PLGA-based formulations other than microspheres and nanoparticles. For example, polymeric micelles and polyplexes are receiving increasing attention as nanocarriers. However, PLGA alone does not permit such formulations.

- PLGA nanoparticles cannot distinguish different cell types. Although cell-recognition capabilities can be conferred to PLGA nanoparticles through conjugation with an appropriate ligand, PLGA itself does not have versatile functional groups that could be used for surface derivatization.

- In vivo blood circulation time of nanoparticles and the extent to which they are taken up by cells are affected by various factors, such as surface charge. For example, cationic nanoparticles enhance cellular uptake and open tight junctions. It is sometimes necessary to change the negative charge of bare PLGA nanoparticles.

- The passive diffusion of PLGA nanoparticles into tumor tissues, driven by the EPR mechanism, may not elicit an efficacious clinical response. Transferring more drug payload to cancer cells is required.

- Bare PLGA nanoparticles do not easily enter cells, and they cannot pass through the blood-brain barrier (BBB). Innovative measures should be taken to improve their uptake by cells or to use them as a drug carrier to the brain.

Versatile strategies are being adopted to overcome the limitations of bare PLGA nanoparticles. The objectives of these strategies are to improve their functionality and in vivo performance. Functionalization of PLGA nanoparticles includes surface modification by PEGylation (PEG, polyethylene glycol), lipid- or surfactant-coating, polyion complexation, and conjugation with cell-targeting ligands. Functional PLGA-based nanoparticulate systems offer various beneficial outcomes, such as: minimization of opsonin adsorption, prolongation of blood residence time, reduction of drug side effects, elimination of the use of toxic adjuvants for drug solubilization, improved targeted drug delivery, efficient cellular translocation and subcellular trafficking, and synergic drug combination therapy. ${ }^{5,6}$ Broadly, functional PLGA-based nanoparticulate forms may be classified as PEGylated micelles, polyplexes, PEGylated nanoparticles, polymersomes, core-shell type hybrid nanoparticles/ nanocells, cell-mimicking nanoparticles, targeting ligandPLGA conjugates, and theranostics (Figure 1). This review focuses on the fundamentals and strategies that are used to develop diverse functional PLGA nanoparticulate carriers. Representative practices for each topic are discussed, rather than specific details.

\section{Why are PLGA and PEG preferred over other polymers?}

The US Food and Drug Administration (FDA) approved the use of PLGA and polylactide (PLA) polymers in parenteral microspheres, implants, and periodontal drug-delivery systems (eg, Lupron Depot, Sandostatin LAR Depot, Zoladex, Vivitrol, Risperdal Consta, OsteoScaf, Arestin, and Atridox). The long approval history of PLA/PLGA-based drug products by worldwide regulatory agencies is a major reason for the preferential use of these biodegradable polymers over others. For convenience, PLGA is used hereinafter to represent both PLA and PLGA. The characteristics of PLGA are controlled by the stereochemistry of lactic acid (D, L, or DL), degree of crystallinity, lactic acid/glycolic acid ratio, and molecular weight. PLGA is available with different end groups, namely a free carboxylic end group and an ester-terminated group. ${ }^{7}$ End-capped PLGA usually has a lauryl or a methyl ester, and is more stable than uncapped PLGA.

PEGylation technology contributes to (a) increasing the aqueous solubility and stability, (b) reducing intermolecular aggregation, (c) decreasing immunogenicity, and (d) prolonging the systemic circulation time of a compound. PEG is often linked to PLGA to achieve similar beneficial effects. ${ }^{8}$ PEG conjugation to PLGA also enables the preparation of selfassembling micelles. In addition, the end groups of PEG can be modified to aldehyde, amino, carboxyl, or methyl groups to alter the surface charge of a nanoparticulate carrier. There are several kinds of PEGs: linear monofunctional PEG derivatives having a reactive group (eg, carboxyl or amine group) on one side and a methoxy group or a sugar group on the other side, homobifunctional PEG derivatives, heterobifunctional PEG derivatives, Y-shape branched PEG derivatives, fork-shaped PEG derivatives, and multi-arm PEG derivatives. ${ }^{9}$ These PEG derivatives are used for conjugation with ligands that have amine, thiol, or carboxyl groups.

\section{PEGylated PLGA micelles}

PEG-PLGA block copolymers are utilized to prepare micelles. ${ }^{10}$ Although various kinds of block copolymers can be synthesized, the most commonly synthesized block 


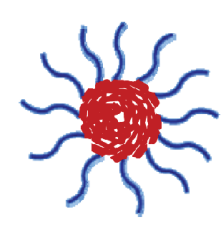

PLGA-PEG block copolymer

A

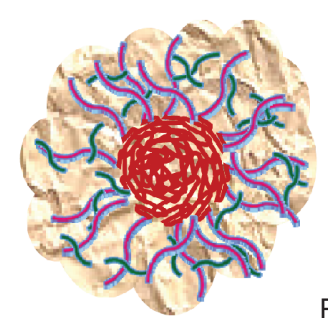

B

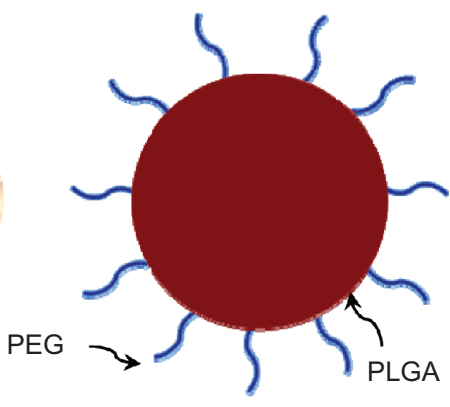

C

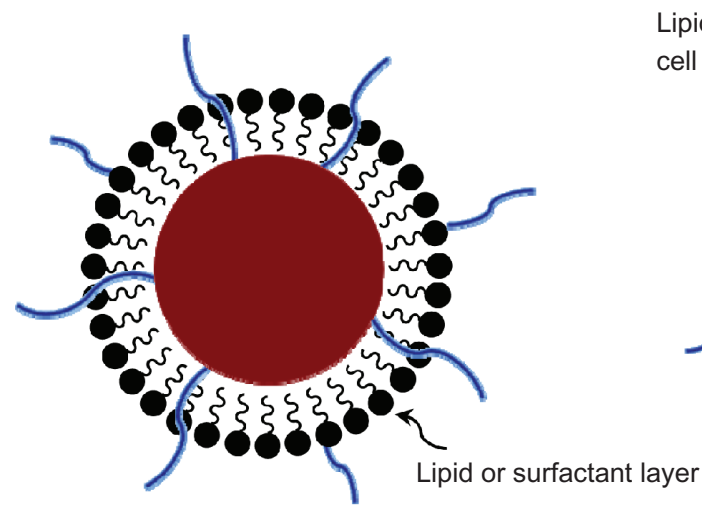

D

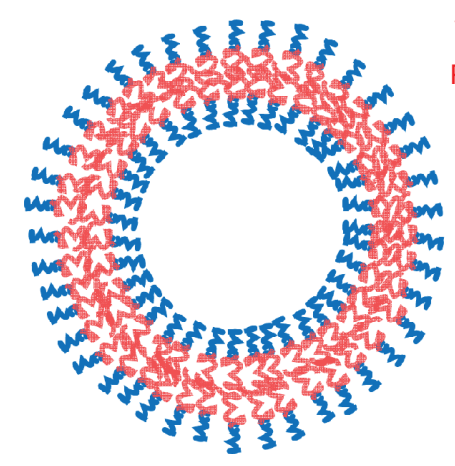

$\mathbf{F}$

Mgan

PLGA-PEG

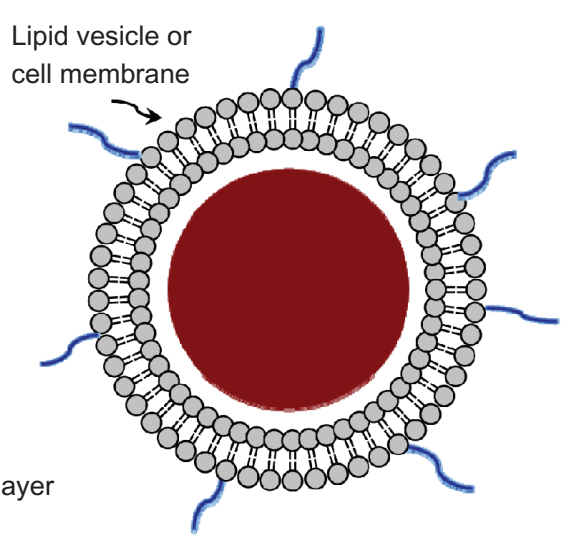

$E$

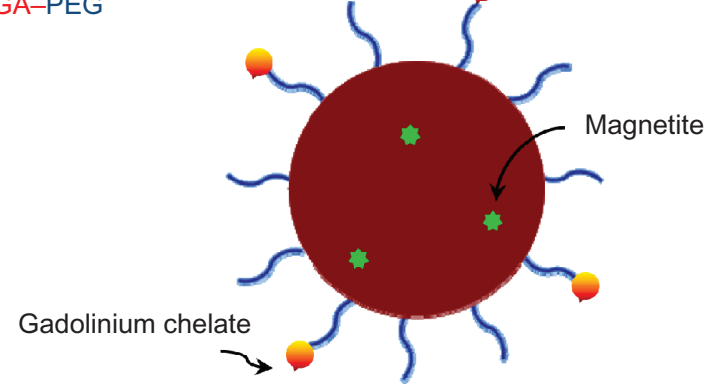

G

Figure I Representative PLGA-based nanoparticulate dosage forms. (A) PEGylated micelle (eg, PEG-PLGA block copolymeric micelle), (B) polyplex (eg, DNA-PEI-PLGA), (C) PEGylated PLGA nanoparticle, (D) core-shell type nanoparticle (eg, PEGylated lipid-PLGA hybrid nanoparticle), (E) cell membrane-PLGA hybrid nanoparticle (eg, PLGA nanoparticles encased by red blood cell), (F) polymersome (eg, PEG-PLGA block copolymeric vesicle), and (G) magnetic PLGA particle either conjugated with gadoliniumchelate or laden with magnetite (eg, theranostics).

Note: All of these nanoparticulate carriers can be further derivatized by cell-recognizable ligands.

Abbreviations: PLGA, polylactide-co-glycolide; PEG, polyethylene glycol; DNA, deoxyribonucleic acid; PEI, polyethyleneimine.

copolymers have $\mathrm{AB}, \mathrm{BAB}$, or $\mathrm{ABA}$ block structures, where A and B stand for PEG and PLGA blocks, respectively. ${ }^{11,12}$ Synthetic methods for producing these copolymers are well established. ${ }^{13-15}$ Currently, a number of block copolymers are commercially available from companies such as Akina (http://www.akinainc.com) and Polysciences, Inc (http://www.polysciences.com). Block copolymers with low molecular weights and/or high PEG/PLGA ratios are water-soluble, whereas those with high molecular weights and/or low PEG/PLGA ratios are water-insoluble. Block copolymers, which are more hydrophilic than bare PLGA, are considered to be more suitable for the delivery of hydrophilic macromolecules such as proteins.

Due to their amphiphilic nature, when PEG-PLGA block copolymers are dispersed in an aqueous medium, they self-assemble into micellar forms. PEG acts as a hydrophilic corona, while PLGA serves as a hydrophobic core. A polymeric micelle can incorporate aqueous 
hydrophobic drugs such as paclitaxel. Polymeric micelles can prolong the blood residence time of drugs, lessen systemic toxicity, and direct drugs to the site of action. ${ }^{16}$ One example of PEG-PLGA micellar technology is the Genexol-PM formulation of Samyang Corporation, Seoul, Korea. ${ }^{17}$ This formulation was approved by the Korea FDA in 2007. Genexol-PM is a Cremophor EL-free PEG-PLGA micelle formulation of paclitaxel that is intended to treat breast cancer and non-small cell lung cancer. This formulation allows administration of higher doses of paclitaxel than the Cremophor EL-based formulation. The maximum tolerated doses of the Genexol-PM and Cremophor EL-containing Taxol formulation were 390 and $230 \mathrm{mg} / \mathrm{m}^{2}$, respectively. ${ }^{18}$ In addition, specialized intravenous infusion sets, in-line filtration, and premedication are not required for the administration of the Genexol-PM. In preclinical models, the Genexol-PM achieved higher paclitaxel tumor concentrations than the Cremophor EL-based formulation. ${ }^{13}$ These results indicate that polymeric micelles may passively target tumors, stemming from the EPR effect and the prolonged systemic circulation time. It may be of interest to the readers to note that Davis et al summarized the pharmacokinetic parameters of nanoparticulate carriers including Genexol-PM, Taxol, Abraxane, Doxil, and other polymer nanoparticles. ${ }^{5}$ Recently, Shin et al carried out an interesting pharmacokinetics study of individual and concurrent delivery of paclitaxel, demethoxygeldanamycin, and rapamycin using aqueous PEGPLGA micellar solutions. ${ }^{19}$ After single drug-loaded, dual drug-loaded, and triple drug-loaded micelles were separately injected via the tail vein of mice, their pharmacokinetic patterns were characterized. The combination of drugs and their dosage affected the extent of pharmacokinetic differences among 1-, 2-, and 3-drug-loaded micelles. Shin et al speculated that these differences might have arisen from alterations of drug-drug interactions, drug metabolism, and micellar stability.

There are many reports of the use of PLGA-based diblock or triblock micelles for solubilizing hydrophobic drugs. ${ }^{20-22}$ The encapsulation of photosensitizers in PEG-PLGA micelles is an example of this solubilization. In photodynamic cancer therapy, photosensitizers react to a specific wavelength of light and produce an activated oxygen form that kills nearby cells. ${ }^{23,24}$ However, photosensitizers also enter healthy tissues, leading to their photosensitization. In addition, many photosensitizers are too hydrophobic for development into an aqueous injectable formulation. As a potential solution for these problems, a photosensitizer (mTHPP; 5,10,15,20-tetrakis (meso hydroxyphenyl)porphyrin) was loaded into PEG-PLGA micelles. ${ }^{25,26}$ The $30 \mathrm{~nm}$ micelles, which contained 2\% mTHPP, entered HN5 head and neck cancer cells and H2009 lung cancer cells. Upon illumination with a $532 \mathrm{~nm}$ laser, the micelles killed the cancer cells.

An interesting approach to controlling the release of drugs from micelles is the use of a mechanical technique. ${ }^{27}$ Hydrophobic nile red was used as a model drug to be encapsulated into PEGylated PLGA micelles. After micellar solubilization, dye release was triggered by high intensity focused ultrasound (HIFU). The strong HIFU-induced cavitation led to the degradation of the diblock copolymer into its constituents, thereby prompting dye release. The authors suggested that the HIFU technique could be utilized as a noncontact and remote control approach to manipulating drug release from polymeric micelles.

The hydrophobicity of PLGA cores can be increased by chemical derivatization. For example, mono-hexyl and di-hexyl substituted lactide monomers have been used to produce hexyl-PLGA via ring-opening polymerization. ${ }^{28,29}$ This technology, owned by SurModics Pharmaceutical Inc, makes it possible to prepare 20-80 nm micelles. PEG-hexylPLGA micelles, due to their elevated hydrophobicity, have greater capacity for loading hydrophobic drugs than do typical PEG-PLGA micelles. For example, the aqueous solubility of cyclosporine A is $0.01 \mathrm{mM}$, but its solubility in PEG-hexyl-PLGA micelles increases to $1.10 \mathrm{mM}^{30}$

\section{Non-PEGylated PLGA micelles}

Polyethyleneimine (PEI) has been widely used as a surfacemodified adsorbent, complex-forming polyelectrolyte, colloidal particle, gene-delivery vehicle, and the like. ${ }^{31,32}$ Attempts have been made to apply PEI to PLGA nanotechnology. ${ }^{33}$ In one study, PEI-PLGA diblock copolymers were prepared according to the conventional dicyclohexylcarbodiimide/ N-hydroxysuccinimide (DCC/NHS) conjugation chemistry. The PEI-PLGA micelles showed better translocation into human keratinocytes than plain PLGA nanoparticles. It is likely that such improvement in cellular uptake arose from the cationic surface of PEI-PLGA micelles and the strong affinity of PEI to lipopolysaccharides and cell membranes, as has been suggested elsewhere. ${ }^{32}$

A poly(L-cysteine)- $b$-PLGA copolymer, linked through a disulfide bond, was formulated into a micellar dosage form. ${ }^{34}$ The hydrophilic polyamino acid domain formed a corona around the hydrophobic PLGA core. The chain lengths of block polymers and the redox status of the disulfide bond affected the size of the polymeric micelles. Other polypeptides made of aspartic acid, glutamic acid, and 
lysine have also been used as coronary segments of PLGAbased micelles. ${ }^{35-37}$ For example, carboxylic end groups of PLGA chains were reacted with primary $\varepsilon$-amino groups of a cationic poly(L-lysine) (PLL), to produce PLGA-g-PLL copolymers of various grafting percentages $(3.6 \%-8.0 \%) .{ }^{37}$ These conjugates self-assembled into $70-80 \mathrm{~nm}$ micelles in water. These polymeric micelles are more thermodynamically stable and have lower critical micelle concentrations than typical surfactant micelles.

Another interesting micellar template consists of hydrophilic siRNA conjugated to PLGA via a disulfide bond. ${ }^{38}$ The amphiphilic conjugate self-assembles in an aqueous environment to form $22 \mathrm{~nm}$ micelles. The net negative charge of the siRNA-PLGA conjugate micelles is nullified by coating with a cationic PEI. The PEI coated siRNA-PLGA micelles exhibited better cellular translocation than typical siRNA-PEI complexes. Another notable feature of siRNA-PLGA conjugate is the disulfide bond between the siRNA and PLGA components. It is expected that the glutathione-laden cells reduce the disulfide bond and release the siRNA from the conjugate. ${ }^{39}$

Because antisense oligonucleotides do not easily enter target cells, a delivery system is required to facilitate cellular uptake. Jeong and Park conjugated a c-myc antisense 15-mer to a carboxyl end group of PLGA via DCC/NHS chemistry. ${ }^{40}$ The resultant amphiphilic oligonucleotide-PLGA conjugate self-assembled into $65 \mathrm{~nm}$ micelles. The endocytosis efficiency of the conjugate was studied in NIH 3 T3 mouse fibroblast cells. The oligonucleotide sparsely entered the cells $(<0.1 \%)$. In sharp contrast, the cellular uptake of the polymeric micelle was significantly greater $(68.3 \%)$. This suggests that these micelles have potential as an antisense carrier.

\section{Polyplexes}

An innovative strategy was used to prepare PEI-PLGA micelle-based polyplexes for gene delivery. ${ }^{41}$ PLGA and branched PEI (bPEI) were linked together to produce PLGA$b$-bPEI- $b$-PLGA by DCC/NHS conjugation chemistry. This polymer was made into $50-60 \mathrm{~nm}$ micelles that had a positive surface charge. The micelles were then condensed with a firefly luciferase receptor gene-encoding plasmid deoxyribonucleic acid (pDNA). In the MCF7 breast cancer cell model, the transfection efficiency of the resulting polyplexes was $10^{3}-10^{4}$ times lower than that of $\mathrm{bPEI} / \mathrm{pDNA}$ complexes. The authors introduced a lower molecular weight bPEI to the micelle/pDNA complexes to induce tighter layerby-layer complexation and augment the cationic surface charge of the polyplexes. The transfection efficiency of the final polyplexes was 50-100 times higher than that of the primary polyplexes. It is also noteworthy that the final polyplexes were lyophilized to powder, whereas typical bPEI/pDNA complexes became sticky after lyophilization.

PLGA-PEG-PLL triblock copolymers have been used to prepare nanoparticulate carriers. PEG imparts a stealth effect upon the PLGA core, whereas PLL confers a cationic charge to the nanoparticles. ${ }^{42}$ The primary $\varepsilon$-amine groups in the lysine moieties of PLL can form polyionic complexes with negatively charged compounds. Such a system was explored as a nonviral vector for DNA transfection. In vitro gene transfection studies using HeLa and HepG2 cells showed that better transfection efficiency was attained with PLGA-PEGPLL/DNA polyplexes than with typical PEI/DNA complexes. Such a system might serve as a safer nonviral gene delivery vector than typical PEI/DNA complexes.

\section{Acid-responsive micelles}

As nanoparticles enter cells by endocytosis, proton pumping into endocytic vesicles is accompanied by a vesicular $\mathrm{pH}$ decrease to 5 . If nanoparticles are responsive to $\mathrm{pH}$ change, this property could be used for intracellular drug targeting. ${ }^{43}$ Based on this strategy, PLGA- $b$-poly(2-ethyl-2-oxazoline)- $b$ PLGA triblock copolymer micelles were prepared to deliver doxorubicin into cancer cells. ${ }^{44}$ The micelles self-assemble into a flower-like morphology - the hydrophilic poly(2-ethyl2-oxazoline) domains form a shell layer over the hydrophobic PLGA core. When poly(2-ethyl-2-oxazoline) $(\mathrm{pKa}=7.1)$ is in an acidic environment, the protonated nitrogen interacts with a carbonyl group via hydrogen-bonding. This triggers the deformation of the polymeric micelles. The $\mathrm{pH}$-dependent destabilization of the micelle structure causes changes in size, zeta potential, fluorescence pattern, and doxorubicin release. A related study on human cervix HeLa cells showed that these polymeric micelles significantly suppressed tumor cells.

\section{Preparation of functional PLGA-based micelles}

Typical methods for preparing polymeric micelles include: (1) the solid dispersion method, (2) the emulsion-based solvent evaporation/extraction method, (3) nanoprecipitation, and (4) the solvent dialysis method. ${ }^{45}$ Suitable manufacturing methods are selected based on the aqueous solubility of the amphiphilic block copolymer, the molecular weight of each block constituent, and the proportion of each block constituent. In the solid dispersion method, an amphiphilic polymer and a hydrophobic drug are dissolved in an organic solvent. The solvent is evaporated under reduced pressure to form a 
gel-like polymeric matrix. Adding water into the gel-like matrix followed by dispersing the mixture at elevated temperature lead to the formation of polymeric micelles (Figure 2A). In the emulsion-based method, a polymeric dispersed phase is emulsified in an aqueous phase. Solvent removal by evaporation and/or extraction causes the rearrangement of polymeric chains to form micelles (Figure 2B). Nanoprecipitation involves the use of a water-miscible solvent (eg, tetrahydrofuran, acetonitrile, acetone, and dimethyl formamide) as a dispersed solvent. Addition of the dispersed phase to an aqueous phase triggers instant solvent diffusion, leading to spontaneous formation of polymeric micelles. Finally, in the solvent dialysis method, a water-miscible solvent is removed by dialyzing with an excessive amount of water.

\section{PEGylated PLGA nanoparticles}

When administered intravenously, bare PLGA nanoparticles are rapidly cleared from the blood. This phenomenon is attributed to the fact that hydrophobic nanoparticles with a negative surface charge are vulnerable to opsonization. ${ }^{46,47}$ To overcome this limitation, the surface of PLGA nanoparticles is layered with PEG that functions as a hydrophilic corona. Improved systemic circulation time of PEGylated PLGA nanoparticles is well documented. ${ }^{48-50}$ PEGylation of PLGA nanoparticles can be conducted in two ways. In one method, nanoparticles are prepared using PEGylated PLGAs such as PEG-PLGA, PLGA-PEG-PLGA, and PEG-PLGA-PEG block copolymers. The other method involves a chemical reaction that links PEG to the surface of preformed PLGA nanoparticles. For example, 1-ethyl3-(3'-dimethylaminopropyl) carbodiimide (EDC)/NHS conjugation results in an amide bond between an amineterminated PEG derivative and a free carboxyl end group of PLGA. Physicochemical properties of PEG-PLGA nanoparticles (size, surface charge, surface hydrophilicity, and surface PEG content) affect their biodistribution and cellular uptake. ${ }^{51,52} \mathrm{Li}$ et al have correlated these nanoparticle properties to biodistribution patterns using a physiologically based pharmacokinetic model. ${ }^{52}$

A comprehensive review of current advances in the stealth coating of nanoparticulate drug-delivery systems was recently published. ${ }^{53}$ The article particularly cautions against the undesirable effects of PEGylation on the in vivo performance of nanoparticulate carriers. The major concerns are the interference of the PEG corona with nanoparticle-cell interactions and the endosomal escape of nanoparticles after extravasation. Another major concern is the immune response of the host to repeated administration of a PEGylated nanoparticulate carrier. It is also important to consider the dependence of stealth properties on the density and morphology of PEG corona. The corona morphology of PEG is often described by mushroom (random coil, low density), brush (elongated coil, high density), or mushroom/ brush transition models. ${ }^{54-56}$ PEG coronas in brush-like and intermediate configurations inhibit phagocytosis and complement activation, whereas mushroom-like PEG morphology triggers complement activation and phagocytosis. Therefore, the molecular weight and shape
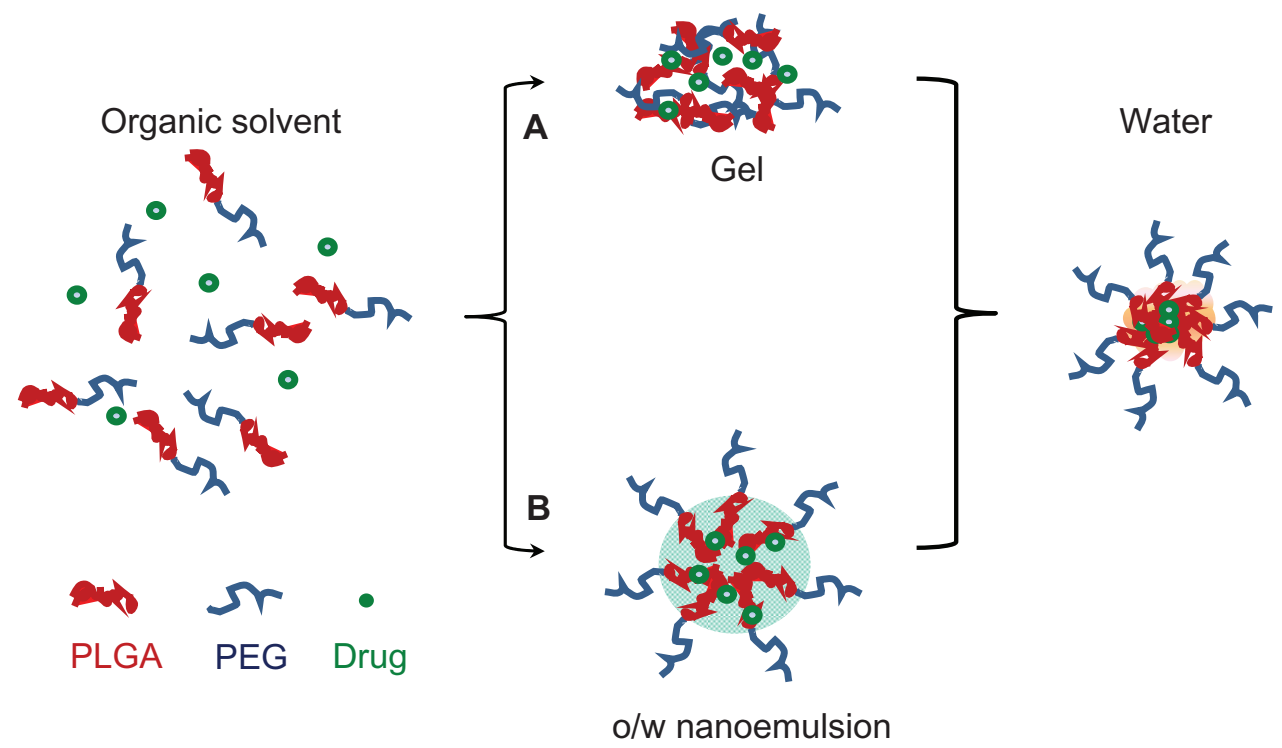

Figure 2 Schematic representation of typical nanoencapsulation techniques used to prepare PLGA-based micelles: (A) the solid dispersion method and (B) the emulsionbased solvent evaporation/extraction method.

Abbreviations: PLGA, polylactide-co-glycolide; PEG, polyethylene glycol; o/w, oil-in-water. 
of PEG, as well as the PEG/PLGA molar ratio, play critical roles in the behavior of PEGylated nanoparticles.

Even though there are a number of nanoencapsulation technologies, the most commonly used technique to prepare PLGA nanoparticles is an emulsion-based solvent evaporation/ extraction technique. ${ }^{57,58}$ The manufacturing principles of this technique are very similar to those used in the production of commercial PLGA microspheres. A water-in-oil-in-water double emulsion technique is used for nanoencapsulation of hydrophilic drugs, whereas an oil-in-water emulsion method is chosen for encapsulation of hydrophobic drugs. Briefly, an organic solvent with substantial volatility (eg, methylene chloride, chloroform, or ethyl acetate) is used to dissolve nanoparticle-forming materials. The dispersed phase is then emulsified in an aqueous phase using a propeller stirrer, a turbine impeller mixer, a rotor-stator homogenizer, a high pressure microfluidizer, a porous membrane mixer, or a sonicator. ${ }^{59,60}$ Solvent removal from nanoemulsion droplets brings about their transformation into solid nanoparticles. Although nanoprecipitation follows the same manufacturing principle as the emulsion-based procedure, the former favors the use of a water-miscible semipolar solvent (eg, acetone, acetonitrile, or tetrahydrofuran) over water-immiscible solvents. As a result, mixing of the polymeric dispersed phase with an aqueous continuous phase leads to instant generation of solid nanoparticles.

\section{Polymersomes}

There are often critical problems with typical liposomes, such as low drug loading efficiency, storage instability, drug leakage during storage, or burst drug release. Polymersomes have therefore been proposed as an alternative to liposomes. ${ }^{61,62}$ Polymersomes are self-assembled bilayer vesicles made from synthetic amphiphilic copolymers such as PEG-PLGA (Figure 1). Polymersomes can be further engineered to deliver, target, and release bioactive materials. ${ }^{63,64}$ Although polymersomes and liposomes are both bilayered vesicles, they differ from one another in several ways. For example, PEGylated lipid content of stealth liposomal formulations is limited to $5 \%-10 \%$, because greater concentrations result in curved micelles ${ }^{65}$ In contrast, polymersomes accommodate much higher concentrations of a PEGylated ingredient, and can be stable for months in aqueous solutions. ${ }^{66}$ The more densely packed PEG brush of polymersomes might confer better resistance toward opsonization than the less densely packed PEG brush of other nanoparticles, thereby extending their systemic circulation time. Both hydrophilic drugs and hydrophobic drugs can be loaded into polymersomes. Drug cocktail therapy using polymersomes has been suggested as a method for directing multiple drugs to multiple cellular targets; for example, taxol to microtubules and doxorubicin to DNA. ${ }^{67,68}$

The self-assembling dynamics of an amphiphile are determined by the characteristics of its constituents and their proportions. The geometry of a self-assembled amphiphile is described by a core packing parameter $(p)$ as follows: $p=v_{\mathrm{H}} /\left(a_{0} \times l_{\mathrm{c}}\right)$, where $v_{\mathrm{H}}$ is the volume of a compactly packed hydrophobic core, $a_{0}$ is the effective cross-sectional area occupied by a hydrophilic group at a nanoparticulatesolution interface, and $l_{\mathrm{c}}$ is the length of a hydrophobic core ${ }^{69}$ Spherical micelles have $P$ value of less than $1 / 3$, and the $P$ value of cylindrical micelles falls between $1 / 3$ and $1 / 2$. Lamellar vesicles have $P$ values ranging from $1 / 2$ to 1 . Many reports prefer to use the $f$ value (the fraction of the hydrophilic block in an amphiphile), rather than the $P$ value. The relationship between $f$ and $p$ is expressed by the following equation: $\ln f \approx-p / \beta$ where $\beta=0.66 .{ }^{61}$ This correlation is helpful to those who are interested in manipulating the morphology of nanoparticulate matters. When a PEG-PLGA block copolymer is used to prepare polymersomes, $f$ values of $0.35 \pm 0.1$ are recommended as a starting point. A copolymer with $f>0.45$ is likely to produce micelles, whereas a copolymer with $f<0.25$ gives rise to solid-like particles. To produce polymersomes, the constituents of a polymersome are dissolved in an organic solvent. After solvent removal, the resultant thin film is hydrated with the aid of heat and/ or a cosolvent. The formation of a bilayered vesicle is favored thermodynamically. Sometimes, polymersomes are sonicated or extruded to tailor their size. They can be further labeled with targeting ligands or fluorescent dyes.

Ester linkages in the backbone of PLGA are subject to random hydrolytic chain scission. This hydrolytic progress influences the vesicular integrity of PEG-PLGA polymersomes and drug release. PLGA hydrolysis transforms the bilayer-forming PEG-PLGA into PEG-abundant segments. These degradation byproducts either segregate to generate hydrophilic pores in the polymersome membrane, or congregate to form detergent-like micelles. ${ }^{70}$ Continual PLGA degradation eventually leads to the rupture of the polymersomic membrane. Accordingly, drug release is degradation-dependent and can range from days to months, depending on polymer characteristics.

\section{Core-shell type hybrid nanoparticles}

Core-shell type lipid-PLGA hybrid nanoparticles usually consist of three major components: (1) drug-loaded PLGA 
nanoparticles, (2) a lipid layer/membrane surrounding the surface of PLGA nanoparticles, and (3) a hydrophilic stealth material such as PEG. ${ }^{71-73}$ PEG is conjugated either to PLGA, or to a lipidic component such as distearoyl phosphatidylethanolamine (DSPE). In addition, a targeting material may be linked to the lipidic material such as lecithin, or conjugated to the surface of nanoparticles. In general, the thickness of the lipid layer on PLGA nanoparticles ranges from 5 to $12 \mathrm{~nm}$. Relevant variables (eg, lipid/PLGA ratio, PLGA property, and lipid type) are changed to prepare coreshell nanoparticles with desired in vivo behavior. ${ }^{74}$ A coreshell type nanoparticle provides better drug encapsulation efficiency than PEG-PLGA nanoparticles or bare PLGA nanoparticles. ${ }^{75}$ It is likely that the lipidic layer acts as a molecular fence that helps to entrap drug molecules inside the hard core of hybrid nanoparticles.

Lipid-PLGA hybrid nanoparticles are prepared by either a single-step procedure or a two-step procedure. ${ }^{76-78}$ In the typical single-step process, PLGA is dissolved in an organic solvent (eg, acetonitrile). Separately, a preheated aqueous solution containing $4 \%-6 \%$ ethanol is used to dissolve lecithin and functionalized lipids (eg, PEG-DSPE). The polymeric solution is added dropwise into the aqueous phase with vigorous mixing to generate lipid-coated nanoparticles. The hydrophobic domains of the lipidic materials adsorb to the surface of PLGA cores, and their hydrophilic domains project toward the aqueous phase. The organic solvent diffuses into the aqueous phase and is removed by dialysis, evaporation, or extraction. In the preparation of lipid-PLGA hybrid nanoparticles, the lipid/PLGA ratio is critical. At a lipid concentration above its critical micelle concentration value, micelles and/or liposomes might coexist with core-shell nanoparticles. In the two-step manufacturing process, PLGA nanoparticles and lipid vesicles are prepared separately. They are then merged together to produce lipid-PLGA hybrid nanoparticles. When a hydrophilic drug is loaded into hybrid nanoparticles, counter ionic excipients (eg, PEG-PE and dextran sulfate) can be included in their formulation. Ionic excipients induce electrostatic interactions with drugs, thereby improving their incorporation efficiency. ${ }^{79-81}$

Lipid-PLGA hybrid nanoparticles enable multiple drug combination therapy and temporal release of more than two drugs. An interesting nanoparticulate delivery system was proposed to target tumor cells and neovasculature at the same time. ${ }^{77}$ The "nanocell" architecture consisted of a nuclear nanoparticle and an extranuclear lecithin/PEGylated lipid envelope, which could be categorized as a core-shell template. More specifically, a doxorubicin-PLGA conjugate was used as a nanoparticle-forming matrix. In a separate experiment, combretastatin A4, which could damage tumor blood vessels, was loaded into liposomes consisting of DSPE-PEG, phosphatidylcholine, and cholesterol. After doxorubicinPLGA nanoparticles were added to the liposome suspension, the mixture was extruded to produce nanocells. In a murine tumor model, combretastatin A4 was released quickly to inhibit the growth of tumor blood vessels. Following the breakdown of the doxorubicin-PLGA conjugate, doxorubicin was slowly released to kill tumor cells.

In the preparation of other core-shell type hybrid nanoparticles, the lipidic shell component can be replaced with other materials, as in a surfactant-PLGA hybrid nanoparticulate system. ${ }^{82,83}$ After surfactant-coated nanoparticles that contained doxorubicin $\mathrm{HCl}$ were injected intravenously into tumor-bearing rats, their survival time was monitored. Animal survival time was considerably longer when rats were injected with nanoparticles coated with polysorbate 80 , TPGS (D- $\alpha$-tocopheryl polyethylene glycol 1000 succinate) or poloxamer 188 than when they were injected with bare doxorubicin-loaded nanoparticles. Another example of core-shell type nanoparticles is a chitosan-PLGA hybrid. Chitosan is cationic and interacts with the anionic components (eg, sialic acid) of glycoproteins on cell membranes. Chitosan is regarded as less toxic than other cationic polymers such as PLL and PEI. It can condense with negatively charged nucleotides, thus improving their transfection efficacy. ${ }^{84,85}$ Based on these properties of chitosan, it has been used to modify the surface of PLGA nanoparticles in order to improve their binding capacity to negatively charged nucleotides and to enhance cellular internalization. ${ }^{86,87}$ Zhao et al used PEGylated octadecyl-quaternized lysine-modified chitosan as a lipid shell for PLGA nanoparticles containing paclitaxel. ${ }^{88}$ The surface of the nanoparticles was further functionalized by the conjugation with folic acid for targeted delivery of paclitaxel. In a study of mice bearing cervix tumors, the functional PLGA nanoparticles provided better antitumor efficacy than Taxol. The use of functional PLGA nanoparticles also resulted in a drug concentration at the tumor site that was ten times that of a paclitaxel solution. The EPR effect and receptor-mediated endocytosis might explain these beneficial outcomes.

\section{Cell-PLGA hybrid nanoparticles}

Encapsulating PLGA nanoparticles into erythrocyte membranes has been studied as a new means of constructing a biomimetic PLGA nanoparticulate delivery system. ${ }^{89}$ Fresh 
red blood cells (RBCs) were incubated in a hypotonic medium to induce hemolysis. This procedure led to RBC ghosts that were devoid of cytoplasmic content. These ghosts were sonicated and extruded through a polycarbonate membrane to produce RBC membrane-derived vesicles. Preformed PLGA nanoparticles were put together with the RBC membranebased vesicles, and the mixture was extruded to fuse them together. The PLGA core was approximately $70 \mathrm{~nm}$ in diameter, and the outer membrane shell was 7-8 nm thick. A related in vitro study demonstrated that the cell-PLGA hybrid nanoparticles entered HeLa cells without being disrupted. A pharmacokinetic study using mice showed that the hybrid nanoparticles had a superior blood retention effect, compared with PEG2000-functionalized lipid-PLGA hybrid nanoparticles (their corresponding elimination halflives were 39.6 and 15.8 hours, respectively). This beneficial effect might have arisen from the higher structural rigidity and better biomimetic property of the RBC membrane, in comparison to PEG2000-functionalized lipid-PLGA hybrid nanoparticles.

\section{Surface derivatization of PLGA nanoparticles}

Functionalization of PLGA nanoparticles can also be achieved by modifying the nanoparticle surface with celltargeting ligands. Attaching the desired ligand to the surface of nanoparticles can be performed by simple physical associations or conjugation reactions (Figure 3). Physical associations are driven by electrostatic interactions and hydrophobic associations, as seen in polyplexes and coreshell type lipid-PLGA hybrid nanoparticles. Specific binding affinity is responsible for the interactions between ligands and biotin-functional PLGA nanoparticles. ${ }^{90}$ For example, preformed PLGA nanoparticles with carboxyl end groups can be conjugated with biotin-PEG- $\mathrm{NH}_{2}$. Avidins (avidin and its homologues such as Streptavidin and NeutrAvidin) show very high affinity to biotin. Therefore, biotinylated PEG-PLGA nanoparticles serve as a platform for further noncovalent binding with avidin-ligand conjugates (Figure 3A). Similarly, biotin-ligand conjugates can be attached to avidin-functionalized PLGA nanoparticles. Targeting ligands (eg, antibodies and Fab fragments) can be attached to PLGA nanoparticles using these simple methods. Such approaches help avoid harsh conjugation chemistry conditions, thereby guaranteeing ligand stability.

The formation of an amide using a carbodiimide is often used to conjugate a ligand to nanoparticles (Figure 3B-E). For example, a water soluble carbodiimide reagent such as
EDC reacts with a carboxyl group in PLGA, forming an amine-reactive $O$-acylisourea intermediate. Although the intermediate reacts with an amine group in the ligand, it is also prone to other reactions. Therefore, NHS is added to transform the intermediate into its NHS ester derivative. The rapid reaction of the ester derivative with any primary amine group and the liberation of NHS lead to the formation of a PLGA-ligand conjugate that is linked through an amide bond. This EDC/NHS conjugation reaction occurs in an aqueous environment. Because some portions of free carboxyl end groups in PLGA are embedded inside PLGA nanoparticulates, their availability for direct conjugation might be limited. To avoid this situation, PLGA is dissolved in an organic solvent such as dimethyl formamide, prior to the conjugation reaction. In this case, EDC is replaced with a hydrophobic carbodiimide reagent (eg, DCC).

The surface of PLGA is often derivatized by various PEG types with different end group functionalities for PEGylation effects and reactions with diverse ligands. At present, amine-, aldehyde-, carboxyl-, maleimide-, succinimidyl ester-, and sulfhydryl-functionalized PEG-PLGA polymers are available. Representative examples of the conjugation methods used for surface derivatization of PLGA nanoparticulate systems are discussed in the relevant sections of this manuscript.

\section{Bisphosphonate-functional PLGA nanoparticles}

Bisphosphonates cause osteoclast apoptosis and are used to increase bone mass and to treat bone metastases. Non-nitrogencontaining bisphosphonates (eg, clodronate and etidronate) are metabolized to nonhydrolyzable analogs of adenosine triphosphate and diadenosine tetraphosphate. In contrast, nitrogen-containing bisphosphonates (eg, zoledronate, pamidronate, ibandronate, and alendronate) are inhibitors of the mevalonate pathway. Bisphosphonates have strong affinity to calcium and tend to accumulate in bones. This property has been utilized to synthesize biphosphonate-PLGA conjugates that can be used as a nanoparticle-forming matrix. ${ }^{91}$ Alendronate and ibandronate have primary amine groups that can be conjugated to the carboxyl end groups of PLGA. For example, an alendronate-PLGA conjugate was used to prepare 200-300 $\mathrm{nm}$ nanoparticles that could be directed to the bones..$^{92}$ Alendronate-PLGA conjugate nanoparticles do not have cytotoxic effects on endothelial cells or trabecular osteoblasts. These nanoparticles adsorb onto hydroxyapatite to a greater extent than bare PLGA nanoparticles.

Bisphosphonates are cytotoxic to tumor cells and macrophages. ${ }^{93,94}$ For example, clodronate encapsulated 
Biotinylated nanoparticle avidin-ligand conjugate

A

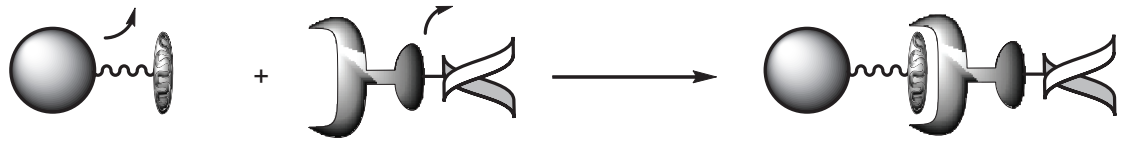

B

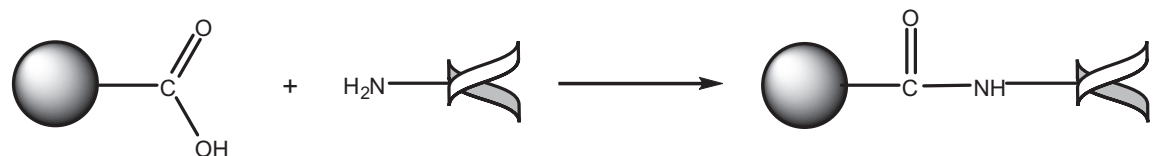

C

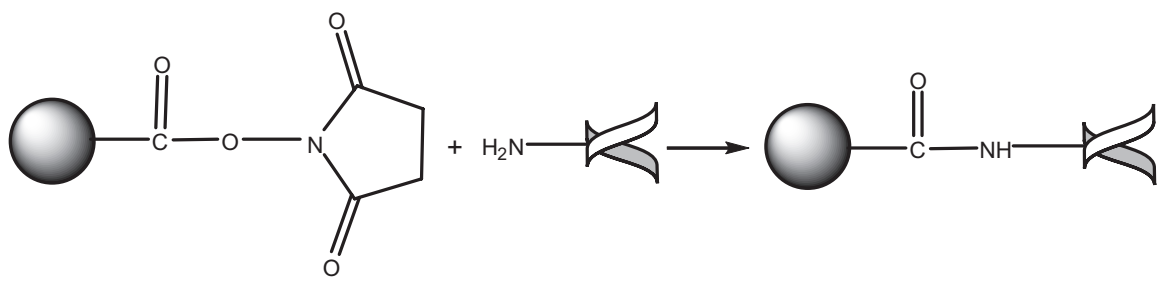

D

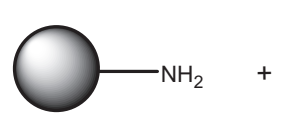<smiles>Cc1ccc(C(=O)O)cc1C</smiles>

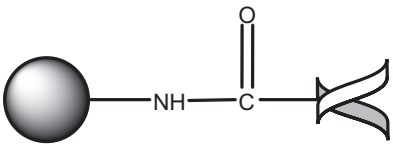

E<smiles>O=C1C=CC(=O)N1c1ccccc1</smiles>

$+$
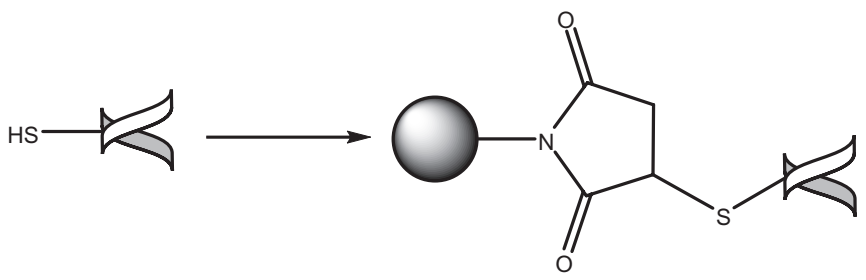

$\mathbf{F}$
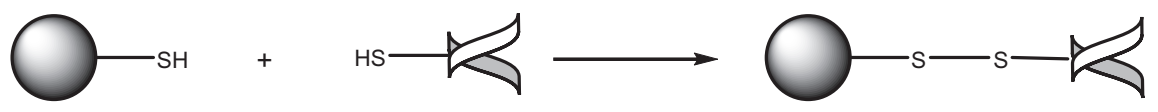

G
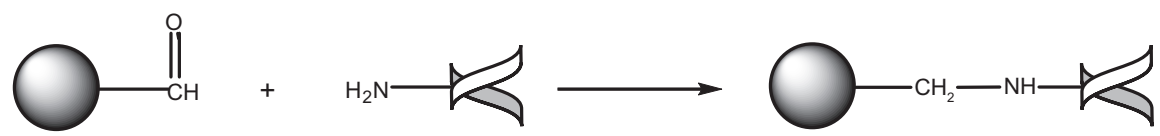

Figure 3 Derivatization of the surface of PLGA-based nanoparticulate carriers with cell-recognizable ligands: (A) physical association driven by the specific avidin-biotin binding affinity; (B-D) amide coupling reactions using carbodiimide reagents; (E) maleimide-thiol reaction; (F) thiol-thiol reaction; and (G) aldehyde-amine reaction. In most conjugation reactions, the surface of PLGA is first derivatized by PEGs with different end groups to produce amine-, aldehyde-, maleimide-, succinimidyl ester-, or sulfhydrylfunctional PEG-PLGA conjugates.

Abbreviations: PLGA, polylactide-co-glycolide; PEG, polyethylene glycol.

in liposomes is reported to be toxic to macrophages, and liposomal alendronate is more potent against cancer cells than free alendronate. Tumor associated macrophages (TAMs) support tumor growth and help existing tumors evade the host immune system. Therefore, the targeting of TAMs is perceived as a promising cancer therapy. Liposomal clodronate is an example of this approach. ${ }^{95,96}$ Similarly, clodronate-containing PLGA nanoparticles have been proposed as a method for reducing macrophage density in tumor patients. ${ }^{97}$ To improve tumor targeting, the surface of the nanoparticles is functionalized by the addition of a tumor-specific peptide, LyP-1 (CGNKRTRGC). An in vivo study using $\mathrm{BALB} / \mathrm{C}$ mice bearing $4 \mathrm{~T} 1$ tumors demonstrated that clodronate-loaded, LyP-1-functionalized PLGA nanoparticles specifically targeted and reduced TAMs. Such studies suggest that bisphosphonate-containing PLGA nanoparticles have potential as a drug carrier for the treatment of bone diseases and cancer immunotherapy.

\section{Lectin-functional PLGA nanoparticles}

Because lectins are capable of recognizing sugar molecules, they show specific binding affinity toward glycosylated membrane components. For example, wheat germ agglutinin, a lectin from Triticum vulgare, is capable of binding to $N$-acetyl- $D$-glucosamine- and sialic acid-containing 
glycoproteins which are abundant in tumors, the intestine, and the nasal cavity. Several lectins have been conjugated with PEG-PLGA nanoparticles to promote cellular uptake. ${ }^{98,99}$ In most cases, lectins were conjugated to maleimidederivatized PEG-PLGA through a maleimide-thiol coupling reaction. Lectin-functional PEG-PLGA nanoparticles bind to various model cells and become endocytosed by clathrinand caveolae-mediated pathways. ${ }^{100}$ In addition, lectinconjugated PEG-PLGA nanoparticles are reported to have potential as a drug carrier for brain targeting. ${ }^{101,102}$

\section{Mannan-decorated PLGA nanoparticles}

C-type lectin receptors (CLRs) that recognize exogenous and endogenous carbohydrates are examples of dendritic cell endocytic receptors. More than 60 human CLRs have been identified. ${ }^{103}$ A mannose receptor that belongs to the CLR family is expressed in subsets of dendritic cells and in macrophages. Mannan-decorated PLGA nanoparticles have therefore been designed to achieve targeted antigen delivery to dendritic cells. ${ }^{104}$ Mannan was either physically adsorbed on the surface of PLGA nanoparticles (139 $\pm 21 \mu \mathrm{g}$ per mg PLGA nanoparticles) or covalently bound via DCC/ NHS conjugation chemistry $(365 \pm 36 \mu \mathrm{g}$ per mg PLGA nanoparticles). A study using bone marrow-derived dendritic cells demonstrated that the cellular uptake efficiency of mannan-decorated PLGA nanoparticles was about twice that of bare PLGA nanoparticles. This result indicates the potential of Mannan-decorated PLGA nanoparticles as vaccine carriers to dendritic cells.

\section{Sialic acid-functional PLGA nanoparticles}

PLGA nanoparticles conjugated with BBB receptor-specific ligands have been used to target the brain. Tosi et al modified the surface of PLGA nanoparticles with a BBB-penetrating peptide (simil-opioid peptide, 7 mer) and sialic acid. ${ }^{105,106}$ Sialic acid was used because of its abundance in the brain parenchyma. While bare PLGA nanoparticles were unable to cross the BBB, the bifunctional PLGA nanoparticles not only provided a targeted brain delivery but also prolonged drug residence time within the brain parenchyma. Although loperamide alone could not penetrate the $\mathrm{BBB}$, the drug-loaded bifunctional PLGA nanoparticles entered the brain and elicited central pharmacological efficacy for more than 24 hours. The duration of drug action attained with the bifunctional PLGA nanoparticles was longer than that attained using other nanoparticulate systems. ${ }^{107,108}$ Biodistribution studies of the bifunctional PLGA nanoparticles revealed that $6 \%$ of the total injected dose of loperamide was localized inside the brain parenchyma of rats. This phenomenon could be attributed to interactions between the bifunctional PLGA nanoparticles and sialic acid receptors in the brain. These exciting preliminary findings indicate that bifunctional nanoparticles can enter the brain and release cargo over an extended period of time.

\section{Biotin-functional PLGA nanoparticles}

Because biotin receptors are overexpressed in many types of cancer cells, it is assumed that decoration of PLGA nanoparticles with biotin enables tumor targeting. Paclitaxel and tariquidar have been coencapsulated into biotin-conjugated PEG-PLGA nanoparticles to target breast cancer cells. ${ }^{109}$ In comparison to biotin-free nanoparticles, the resultant nanoparticles improved the degree of tumor reduction and the survival rate of tumor-bearing mice. Recently, Patil et al have proposed an interesting strategy to overcome multidrug resistance. ${ }^{110}$ Both paclitaxel and a P-glycoprotein-targeted siRNA were coencapsulated into biotin-functional PLGA nanoparticles to maximize antitumor efficacy. An in vivo study of BALB/c mice bearing tumors led to the conclusion that dual drugs containing functional nanoparticles were more effective in reducing tumor volume in mice than control treatments. Concurrent delivery of chemosensitizers and chemotherapeutics via tumor-targeted nanoparticles may be an effective practice in solving multidrug resistance of tumors.

\section{Folate-functional PLGA nanoparticulates}

Many types of cancer cells overexpress folate receptors by as much as 100-300 times the rate of normal cells. ${ }^{11}$ Yoo and Park used a folate receptor for targeting nanoparticulate doxorubicin micelles. ${ }^{12}$ In their study, PLGA was reacted with PEG-bisamine to produce a PLGA-PEG-NH ${ }_{2}$ diblock copolymer. Folate was conjugated to this diblock copolymer via carbodiimide chemistry, and the conjugate was used to prepare doxorubicin-containing micelles. The folatefunctional micelles were more cytotoxic to the human squamous carcinoma cell line of oral cavity ( $\mathrm{KB}$ cells) than controls. The half-maximal inhibitory concentration values of the folate-functional micelles, doxorubicin micelles, and free doxorubicin were about 50,70 , and $75 \mu \mathrm{M}$, respectively. In an in vivo mouse study, the folate-functional micelles caused more significant suppression of tumor growth among 
various doxorubicin preparations. The increase of cellular uptake may have been due to the folate receptor-mediated endocytosis mechanism. Another recent study resulted in the same conclusion. ${ }^{113}$ Wang and Hsiue linked folate and PLGA to PEI diamine, to produce a folate-PEI- $b$-PLGA conjugate. ${ }^{114}$ This amphiphilic block copolymer was able to condense a plasmid DNA (luciferase-encoding pUHC 13-3) to form micellar polyplexes. The folate-PEI- $b$-PLGA polyplexes reduced the degree of luciferase expression. Another folatereceptor targeted nanoparticle was more readily translocated into folate receptor positive cancer cells (SKOV3) than bare nanoparticles. ${ }^{115}$ Polymeric nanoparticles with surfaceexposed folic acid have the potential to be rapidly cleared from systemic circulation due to their immediate interactions with receptors that are present in both cancer and normal cells. To minimize their premature elimination, folatepoly(amidoamine) dendrimer conjugates were encapsulated into PEG-PLGA nanoparticles. ${ }^{116}$ The EPR mechanism helps these nanoparticles accumulate at the targeted tumor. Once released at the tumor site, the dendrimers (12.1-19.6 nm) are likely to enter the tumor cells more effectively than larger nanoparticles (97.6-146.9 nm). The release kinetics of the dendrimers may be further controlled by altering the PEGPLGA properties.

\section{Transferrin-functional PLGA nanoparticles}

Transferrin is a glycoprotein that has a molecular weight of $80 \mathrm{kDa}$. Transferrin receptors are dimeric transmembrane glycoproteins that are overexpressed in many types of tumor cells. ${ }^{117}$ Many research groups have therefore anchored transferrin to PLGA-based nanoparticles. ${ }^{118,119}$ As an alternative to direct linking of transferrin to PLGA nanoparticles, Zheng et al prepared a transferrinDOPE (1,2-dioleoyl-sn-glycero-3-phosphoethanolamine) conjugate. ${ }^{120}$ They used the conjugate as a component of coreshell type lipid-PLGA hybrid nanoparticles that contained an aromatase inhibitor. An in vitro aromatase inhibition test on SKBR-3 cells showed that the activity of transferrin receptortargeted nanoparticles was two times that of transferrin-free nanoparticles. Other studies have also demonstrated the receptor-mediated improved cellular uptake of transferrincoupled liposomes and lipid nanoparticles. ${ }^{121,122}$

\section{Peptide-functional PLGA nanoparticulates}

Integrins are heterodimeric transmembrane subunits of $\alpha$ and $\beta$ that have specific affinities toward peptides with an arginine-glycine-aspartate (RGD) sequence. Among the numerous kinds of RGD, cyclic RGD (cRGD) peptides have better cell attachment than the corresponding linear RGD peptides. ${ }^{123}$ A number of cRGD peptides are currently available for drug-delivery systems and imaging tools for tumors. For example, PLGA-four-arm-PEG branched nanoparticles conjugated to cRGD were developed to target pancreatic tumors. ${ }^{124}$ These functional nanoparticles entered U87MG glioma cells that overexpressed the $\alpha v \beta 3$ integrin. An in vivo study demonstrated that more cRGD-functional nanoparticles accumulated at the pancreatic tumor site than did other controls. Toti et al conjugated a cRGD peptide to maleimide-derivatized PLGA nanoparticles to target $\alpha v \beta 3$ integrins that were overexpressed in tumor vasculature and tumor cells. ${ }^{125}$ A mouse 4T1 tumor model study demonstrated that the cRGD-based functionalization contributed to a nearly twofold increase in the accumulation and retention of the functional nanoparticles in the tumor tissue, as compared with bare nanoparticles. Other RGD-based approaches include creation of new copolymeric materials such as poly(lactideco-lysine) and simple coating of polymeric surfaces with PLL-RGD peptide conjugates. ${ }^{126,127}$

Cell-penetrating peptides (CPPs) have high translocational activity and facilitate cellular uptakes of large molecules and nanoparticulates. ${ }^{128,129}$ Their membrane translocation is mediated by different pathways including direct penetration, endocytosis, and formation of a transitory structure. Trans-activating transcriptional activators (TATs) are examples of CPPs. Several TATs were conjugated to PLGA nanoparticulate systems to direct anticancer agents to tumors and to achieve brain drug delivery. ${ }^{130,131}$ For example, Yu et al synthesized the aldehyde-PEG-PLGA block copolymer, which was used to prepare nanoparticles loaded with paclitaxel. ${ }^{130}$ Using the N-terminal PEGylation technique, a K237 peptide (HTMYYHHYQHHL) was linked to the aldehyde group of PLGA-PEG nanoparticles. (The K237 peptide binds specifically to the kinase insert domain receptor [KDR] receptors that were predominantly expressed on the surface of tumor neovasculature, thereby suppressing the vascular endothelial growth factor [VEGF]-KDR angiogenic signal pathway.) The K237-conjugated nanoparticles displayed improved translocation into human umbilical vein endothelial cells (HUVEC) through K237-KDR interaction, resulting in enhanced paclitaxel activity. Nam et al reported a similar result with a TAT peptide that was conjugated to PLGA nanoparticles. ${ }^{132}$

A bifunctional PEG-PLGA nanoparticulate system containing vincristine sulfate has been recently proposed 
for use in place of monofunctional CPP-conjugated PLGA nanoparticles. ${ }^{133}$ In this approach, both folic acid and a

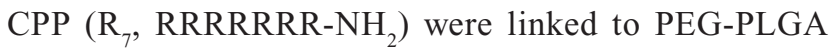
nanoparticles. However, there was only a marginal increase in the cellular uptake of these nanoparticles by MCF-7 cancer cells, compared with PEG-PLGA nanoparticles decorated with either folic acid or $\mathrm{R}_{7}$ alone.

It is worth describing an example of the utilization of the pathophysiology of tumors or ischemic cardiac cells for drug targeting. Their hypoxic conditions lead to the accumulation of lactic acid, thereby generating an acidic environment. Acidsensitive PLGA-based nanoparticulate drug-delivery systems have been designed based on this idea. One example of such systems is a multifunctional micellar system that consists of TAT-PEG-PLGA and a pH-sensitive diblock copolymer, poly(L-cystine bisamide- $g$-sulfadiazine)- $b$-PEG (PCBS-bPEG). ${ }^{134}$ At normal $\mathrm{pH}$, the negatively charged sulfadiazine in the PCBS- $b$-PEG associates with the cationic TAT that is linked to the PEG-PLGA. This electrostatic interaction triggers the shielding of TAT domains. Because only PEG is present on the surface of the micellar polyplexes, their systemic circulation is extended. At an acidic tumor site, the nanoparticles are subject to a change in the charge of sulfonamide, which in turn destroys the physical association between sulfonamide and TAT. This deshielding leads to TATmediated translocation of the TAT-PEG-PLGA micelles into tumor cells. On the other hand, the detached PCBS- $b$-PEG is degraded by glutathione.

A different kind of peptide has also been used for PLGA functionalization. Intracellular cell-adhesion molecules (ICAM) are examples of cell adhesion molecules that are involved in cell trafficking and proliferation of various metastatic cancer cells. For example, ICAM-1 is overexpressed in many diseases such as atherosclerosis, ischemia, asthma, arthritis, and cancer metastasis. ${ }^{135}$ Linear LABL and cyclic LABL (cLABL) peptides (eg, ITDGEATDSG, cyclo-(1,12)PenITDGEATDSGC) bind effectively to ICAM-1 and inhibit its activity. Furthermore, these peptides undergo cellular translocation that is mediated by ICAM-1. Chittasupho et al made use of this mechanism to target doxorubicin-loaded PLGA nanoparticles to lung epithelial cells. ${ }^{136}$ The surface of the doxorubicin-loaded PLGA nanoparticles was coated with carboxyl group-modified Pluronic F127 using a simple adsorption process. The amine group of cLABL was reacted with the carboxyl group of Pluronic F127 via EDC/NHS conjugation chemistry. Cellular uptake studies using A549 lung epithelial cells showed that the binding and uptake of the cLABL-PLGA conjugate nanoparticles were considerably more rapid than those of bare nanoparticles. Zhang et al showed that LABL-functionalized PLGA nanoparticles were more selective for HUVEC cells than plain PLGA nanoparticles. $^{137}$

\section{Antibody-directed PLGA immunonanoparticles}

To improve the targeting efficiency of PEGylated PLGA nanoparticles to a tumor site, the surface of the nanoparticles is often conjugated with monoclonal antibodies (mAb) against overexpressed or lineage-restricted tumor-specific antigens. Examples of $\mathrm{mAb}$ linked to PLGA nanoparticles include antiHer2, anti-ICAM-1, anti-epithelial cell adhesion molecule, anti-DR5, and anti-cytokeratin. ${ }^{138-144}$ For example, DR5 is overexpressed in colorectal tumor cells. ${ }^{144}$ Conatumumab is a mAb that specifically binds to the extracellular domains of DR5 and agonizes the DR5 receptor to trigger apoptotic cell death. Fay et al linked conatumumab to camptothecin-loaded PLGA nanoparticles, in order to target DR5-expressing HCT116 cells. ${ }^{142}$ In parallel, the authors explored the chemotherapeutic effect of camptothecin and the apoptosis-inducing capability of immunonanoparticles. Using EDC/NHS conjugation, $\mathrm{mAb}$ was linked to preformed camptothecin-containing nanoparticles. An in vitro HCT116 cell study indicated that the immunonanoparticles exhibited specific cell-surface binding and receptor-induced internalization. The immunonanoparticles provoked the DR5-caspase 8 apoptotic process and potentiated the chemotherapeutic effect of camptothecin to a greater extent than mAb-free PLGA nanoparticles. Similarly, a cell-specific and targeted delivery was achieved using anti-Fas targeted camptothecin-containing PLGA nanoparticles. ${ }^{145}$

The success of PLGA immunonanoparticles often depends on how well an $\mathrm{mAb}$ is engineered onto the surface of nanoparticles. For example, Obemajer et al loaded cystatin into PLGA nanoparticles and modified the surface of the nanoparticles with anti-cytokeratin $\mathrm{mAb}$ to target cytokeratin on the membrane of breast tumor cells. ${ }^{143}$ The mAb was attached to the nanoparticles by either physical adsorption or EDC conjugation. The $\mathrm{mAb}$ adsorbed nanoparticles entered MCF neoT tumor cells and released cystatin, whereas the same nanoparticles did not pass through noncancerous Caco-2 cells. In contrast, the mAb-nanoparticle conjugates produced via EDC conjugation displayed poor cellular uptake efficiency. It is probable that EDC conjugation damaged the structural integrity of the mAb. Earlier, Kocbek et al reported negative effects of EDC conjugation chemistry on the binding affinity of a mAb. ${ }^{146}$ Similarly, it is suggested elsewhere 
that direct coupling of a targeting moiety to the surface of nanoparticles results in poor binding to the intended receptor and, thus, a poor therapeutic outcome. ${ }^{147}$

The applications of PLGA immunonanoparticles are not limited to cancer therapy. For example, transferrin and insulin receptors are abundant in the brain capillary endothelium..$^{148}$ Brain-targeting PEGylated PLGA immunonanoparticles have therefore been proposed. In one study based on this rationale, the ends of PEG strands in PEG-PLGA block copolymers were functionalized with maleimide, which was used in the fabrication of nanoparticles. ${ }^{149}$ Thiolated mouse OX26 anti-rat transferrin receptor $\mathrm{mAb}$ was then conjugated to the reactive nanoparticles. A transmission electron microscopy evaluation of the immunonanoparticles labeled with anti-mouse IgG antibody-gold conjugate demonstrated that 67 antibodies were conjugated to one nanoparticle. In Alzheimer's disease, amyloid $\beta(\mathrm{A} \beta)$ proteins accumulate in the cerebral vasculature, and cause cerebral angiopathy. This pathophysiological feature was considered in the development of a carrier that could permeate the BBB and target $A \beta$ proteins. Chitosan-coated PLGA nanoparticles conjugated with an anti-A $\beta$ antibody were developed to meet this challenge. ${ }^{150}$ The immunonanoparticles penetrated the BBB and improved targeting to the amyloid proteins. Another application of PLGA immunonanoparticles is the targeting of dendritic cells that are involved in the activation of T-cell-mediated immunity. ${ }^{151,152}$ To accomplish this, PLGA nanoparticles were linked to dendritic cell-specific antibodies including anti-CD11c, anti-CD40, Fc $\gamma, \alpha v \beta 3$, and $\alpha v \beta 5$ integrin receptors. Compared with bare PLGA particles, these immunonanoparticles more effectively induce the maturation of dendritic cells.

\section{Nucleotide-functional PLGA nanoparticles}

Nucleotide-based therapies usually involve three kinds of technologies that make use of antinucleotides, RNA interference, and aptamers. In particular, aptamers are widely used for cancer targets including extracellular ligands and cell-specific proteins. ${ }^{153}$ Aptamers are relatively short DNAor RNA-based oligonucleotides that have 25-40 nucleotides. They possess sequence-specific three-dimensional structures that bind to and inhibit specific target molecules. Macugen, a synthetic PEGylated oligonucleotide that specifically binds to VEGF, is the first such product approved for use in the United States. ${ }^{154}$ However, nucleic acids have several weaknesses, including short half-lives and poor cellular translocation efficiency. To overcome these challenges, aptamers are frequently used to functionalize PLGA nanoparticles for targeted cancer therapy. For example, AS1411 (a 28-bp DNA aptamer) binds to nucleolin, which is highly expressed in the plasma membranes of cancer cells and endothelial cells in angiogenic blood vessels. ${ }^{155}$ Nucleolin is involved in endocytosis and/or macropinocytosis, and it could serve as a targeting ligand for tracking C6 glioma cells. ${ }^{156,157}$ Guo et al therefore evaluated the potential of the AS1411nucleolin interaction for effective drug delivery to gliomas. ${ }^{158}$ Specifically, the AS1411 DNA aptamer was conjugated to the carboxyl end groups of PEG-PLGA nanoparticles that contained paclitaxel. In a rat intracranial tumor model, treatment with the functional nanoparticles increased the animal survival time by 4,7 , and 13 days over treatment with bare nanoparticles, Taxol, and saline, respectively. These results demonstrate the potential of AS1411-nucleolin mediated recognition and internalization of aptamer-functional nanoparticles into C6 glioma cells. AS1411 anti-nucleolin aptamers have also been conjugated to PEGylated lipid-PLGA hybrid nanoparticles containing paclitaxel to target tumor cells that overexpressed nucleolin receptors. ${ }^{159}$ These functional lipid-PLGA hybrid nanoparticles displayed greater cytotoxic effects toward cancer cell lines such as MCF-7 cells than did the aptamer-free nanoparticles. Additional notable features were that this approach enabled better drug encapsulation efficiency and superior sustained drug release than was possible with bare paclitaxel-loaded PLGA nanoparticles. Another example of the use of aptamers for nucleotide-based therapy is the A10 2'-fluoropyrimidine RNA aptamer which displays specific binding affinity to the extracellular domain of a prostate-specific membrane antigen (PSMA). ${ }^{160,161}$ The aptamer-PEG-PLGA conjugate nanoparticles provide a targeted drug delivery (eg, docetaxel) to the prostate cancer cells, thereby effectively inhibiting the proliferation of tumor cells. Similarly, cellular uptake of PLGA-PEG-COOH nanoparticles that were conjugated with a $3^{\prime}-\mathrm{NH}_{2}$-modified 5 -FITC-labeled PSMA aptamer was seven times that of bare PEGylated PLGA nanoparticles. ${ }^{162}$

\section{Magnetic nanoparticles}

A magnetic resonance imaging (MRI)-assisted strategy can improve image resolution at a local site of interest and enable drug delivery to the targeted site. Paramagnetic gadolinium chelates and superparamagnetic iron oxide particles are two main classes of clinically useful MRI contrast agents. Wang et al fabricated $100 \mathrm{~nm}$ poly(D,L-lactic acid-co- $\alpha, \beta$-malic acid) nanoparticles that contained about $8 \%$ magnetite. ${ }^{163}$ The superparamagnetic nanoparticles 
labeled human mesenchymal cells in vitro without damaging their ability to proliferate. Lim et al formulated PLGA nanoparticles that contained magnetite cores. ${ }^{164}$ Because free gadolinium ions are toxic, a chelation strategy is used in the design of magnetic PLGA nanoparticles. For example, a PLGA-PEG block copolymer conjugated with diethylene triamino pentaacetic acid (DTPA) was used as a nanoparticleforming material, and gadolinium was chelated to DTPA that protruded outside the nanoparticles. ${ }^{165}$ An in vivo study demonstrated that the liver-targeting efficiency of gadolinium-labeled PLGA-PEG-DTPA nanoparticles was 14.6 times greater than that of Magnevist, a commercial contrast agent. Using another approach, gadolinium-DTPA was encapsulated into PLGA nanoparticles by a water-in-oilin-oil emulsion technique. ${ }^{166}$ The functionality of magnetic PLGA nanoparticles can be further augmented by the use of additional targeting ligands such as Arg peptides, cLABLs, and antibodies. ${ }^{167,168}$ Advancements in these technologies aid the development of theranostics, which combine biomedical imaging techniques with drug therapies into a single utility.

\section{Conclusion}

A polymeric architecture that is based on PEGylation and polyion complexation has evolved into promising nanocarriers, including self-assembling micelles and polyplexes. Novel biomimetic strategies have helped the emergence of core-shell type hybrid nanoparticles, cell-mimicking nanoparticles, and polymersomes. These nanoparticulate carrier systems have been further functionalized by cell-recognizable targeting ligands (eg, mAbs and their fragments, peptides, DNA/RNA aptamers, and imaging agents). Targeting strategies for these systems usually involve the receptor-mediated cell recognition, endocytosis of nanoparticulate matters, and intracellular drug release. In addition, these functional PLGA nanoparticulate dosage forms provide extended blood residence time, improved EPR effects, multiple drug combination therapies, and better cellular translocation, compared with bare PLGA nanoparticulates. These advantages of functional PLGA nanoparticulate dosage forms have been supported by in vitro cellular uptake and cytotoxicity studies, or by animal and xenograft models. In contrast, some reports indicate that the benefits of these functional PLGA-based nanoparticulate carriers are marginal compared with the corresponding bare PLGA nanoparticulates. In addition, the complete characterization of in vivo behavior of these functional nanoparticulates is still lacking. More systematic studies should be conducted to determine the behavior and stability of these nanoparticulates in the blood. Their pharmacokinetics, drug determination (free drugs versus those entrapped in a nanocarrier), subcellular trafficking, and intracellular dissociation of drugs from nanoparticulate carriers should also be investigated further. Even more surprisingly, there are few studies of the stability kinetics of drug-PLGA and targeting ligandPLGA conjugate nanoparticles. More in-depth investigation is required to ensure that after functional PLGA-based nanoparticulate carriers enter target cells, the drug is released at a rate appropriate to its mechanism of action. Finally, being sought in recent research trends are multifunctional PLGA nanoparticulates that provide temporal drug delivery, enable imaging and drug targeting in a single utility, and achieve synergistic therapeutic outcomes. Advancements in this field would make it possible to develop highly effective nanoparticulate carriers that can deliver a spectrum of chemotherapeutic, diagnostic, and imaging agents for various applications.

\section{Acknowledgments}

This study was supported by a grant from the Korean Health Technology R\&D Project, Ministry for Health, Welfare and Family Affairs, Republic of Korea (A092018).

\section{Disclosure}

The authors report no conflicts of interest in this work.

\section{References}

1. Panyama J, Labhasetwar V. Biodegradable nanoparticles for drug and gene delivery to cells and tissue. Adv Drug Deliv Rev. 2003;55(3):329-347.

2. Danhier F, Ansorena E, Silva JM, Coco R, Breton AL, Préat V. PLGA-based nanoparticles: An overview of biomedical applications. J Control Release. 2012;161(2):505-522.

3. Hobbs SK, Monsky WL, Yuan F, et al. Regulation of transport pathways in tumor vessels: role of tumor type and microenvironment. Proc Natl Acad Sci U S A. 1998;95(8):4607-4612.

4. Maeda H, Wu J, Sawa T, Matsumura Y, Hori K. Tumor vascular permeability and the EPR effect in macromolecular therapeutics: a review. J Control Release. 2000;65(1-2):271-284.

5. Davis ME, Chen ZG, Shin DM. Nanoparticle therapeutics: an emerging treatment modality for cancer. Nat Rev Drug Discov. 2008;7(9): 771-782.

6. Peer D, Karp JM, Hong S, Farokhzard OC, Margalit R, Langer R. Nanocarriers as an emerging platform for cancer therapy. Nat Nanotechnol. 2007;2(12):751-760.

7. Houchin ML, Topp EM. Chemical degradation of peptides and proteins in PLGA: A review of reactions and mechanisms. J Pharm Sci. 2008;97(7): 2395-2404.

8. Vonarbourg A, Passirani C, Saulnier P, Benoit JP. Parameters influencing the stealthiness of colloidal drug delivery systems. Biomaterials. 2006;27(24):4356-4373.

9. Wonganan P, Croyle MA. PEGylated adenoviruses: from mice to monkeys. Viruses. 2010;2(2):468-502. 
10. Yasugi K, Nagasaki Y, Kato M, Kataoka K. Preparation and characterization of polymer micelles from poly(ethylene glycol)poly(d,l-lactide) block copolymers as potential drug carrier. J Control Release. 1999;62(1-2):89-100.

11. Beletsi A, Leontiadis L, Klepetsanis P, Ithakissios DS, Avgoustakis K. Effect of preparative variables on the properties of poly(dl-lactideco-glycolide)-methoxypoly(ethyleneglycol) copolymers related to their application in controlled drug delivery. Int J Pharm. 1999;182(2):187-197.

12. Kissel T, Li Y, Unger F. ABA-triblock copolymers from biodegradable polyester A-blocks and hydrophilic poly(ethylene oxide) B-blocks as a candidate for in situ forming hydrogel delivery systems for proteins. Adv Drug Deliv Rev. 2002;54(1):99-134.

13. Kim SC, Kim DW, Shim YH, et al. In vivo evaluation of polymeric micellar paclitaxel formulation: toxicity and efficacy. J Control Release. 2001;72(1-3):191-202.

14. Ghahremankhani AA, Dorkoosh F, Dinarvand R. PLGA-PEG-PLGA triblock copolymers as an in-situ gel forming system for calcitonin delivery. Polym Bull. 2007;59(5):637-646.

15. Li Y, Pei Y, Zhang X, et al. PEGylated PLGA nanoparticles as protein carriers: synthesis, preparation and biodistribution in rats. $J$ Control Release. 2001;71(2):203-211.

16. Savic R, Luo L, Eisenberg A, Maysinger D. Micellar nanocontainers distribute to defined cytoplasmic organelles. Science. 2003;300(5619): 615-618.

17. Seo MH, Kim BO, Choi IJ, Shim JS, inventors; Samyang Corporation, assignee. Biodegradable branched polylactide derivatives capable of forming polymeric micelles, and their preparation method and use. United States patent US 8021652. September 20, 2011.

18. Kim TY, Kim DW, Chung JY, et al. Phase I and pharmacokinetic study of Genexol-PM, a cremophor-free, polymeric micelle-formulated paclitaxel, in patients with advanced malignancies. Clin Cancer Res. 2004;10(11):3708-3716.

19. Shin HC, Cho H, Lai TC, Kozak KR, Kolesar JM, Kwon GS. Pharmacokinetic study of 3-in-1 poly(ethylene glycol)-blockpoly(D,L-lactic acid) micelles carrying paclitaxel, 17-allylamino-17demethoxygeldanamycin, and rapamycin. J Control Release. 2012; 163(1):93-99.

20. Sutton D, Nasongkla N, Blanco E, Gao J. Functionalized micellar systems for cancer targeted drug delivery. Pharm Res. 2007;24(6): 1029-1046.

21. Song Z, Feng R, Sun M, et al. Curcumin-loaded PLGA-PEG-PLGA triblock copolymeric micelles: preparation, pharmacokinetics and distribution in vivo. J Colloid Interface Sci. 2011;354(1):116-123.

22. Kwon GS, inventor; Wisconsin Alumni Research Foundation, assignee. Micelle encapsulation of therapeutic agents. United States patent application 20120276171. November 1, 2012.

23. Dolmans DE, Fukumura D, Jain RK. Photodynamic therapy for cancer. Nat Rev Cancer. 2003;3(5):380-387.

24. Vrouenraets MB, Visser GW, Snow GB, van Dongen GA. Basic principles, applications in oncology and improved selectivity of photodynamic therapy. Anticancer Res. 2003;23(1B):505-522.

25. Ding H, Mora R, Gao J, Sumer BD. Characterization and optimization of mTHPP nanoparticles for photodynamic therapy of head and neck cancer. Otolaryngol Head Neck Surg. 2011;145(4):612-617.

26. Cohen EM, Ding H, Kessinger CW, Khemtong C, Gao J, Sumer BD. Polymeric micelle nanoparticles for photodynamic treatment of head and neck cancer cells. Otolaryngol Head Neck Surg. 2010;143(1):109-115.

27. Zhang H, Xia H, Wang J, Li Y. High intensity focused ultrasoundresponsive release behavior of PLA-b-PEG copolymer micelles. J Control Release. 2009;139(1):31-39.

28. Trimaille T, Gurny R, Möller M. Synthesis and properties of novel poly(hexyl-substituted lactides) for pharmaceutical applications. Chimia. 2005;59(6):348-352.

29. Trimaille T, Gurny R, Möller M. Poly(hexyl-substituted lactides): novel injectable hydrophobic drug delivery systems. J Biomed Mater Res A. 2007;80(1):55-65.
30. Kasimova AO, Pavan GM, Danani A, et al. Validation of a novel molecular dynamics simulation approach for lipophilic drug incorporation into polymer micelles. J Phys Chem B. 2012;116(14):4338-4345.

31. Helander IM, Alakomi HL, Latva-Kala K, Koski P. Polyethyleneimine is an effective permeabilizer of Gram-negative bacteria. Microbiology. 1997;143(Pt 10):3193-3199.

32. Kirchelis R, Wightman L, Wagner E. Design and gene delivery activity of modified polyethyleneimines. Adv Drug Deliv Rev. 2001;53(3): 341-358.

33. Nam YS, Kang HS, Park JY, Park TG, Han SH, Chang IS. New micelle-like polymer aggregates made from PEI-PLGA diblock copolymers: micellar characteristics and cellular uptake. Biomaterials. 2003;24(12):2053-2059.

34. Sun J, Chen X, Lu T, et al. Formation of reversible shell cross-linked micelles from the biodegradable amphiphilic diblock copolymer poly(L-cysteine)-block-poly(L-lactide). Langmuir. 2008;24(18): 10099-10106.

35. Arimura H, Ohya Y, Ouchi T. Formation of core-shell type biodegradable polymeric micelles from amphiphilic poly(aspartic acid)-blockpolylactide diblock copolymer. Biomaterials. 2005;6(2):720-725.

36. Sun J, Deng C, Chen X, et al. Self-assembly of polypeptide-containing ABC-type triblock copolymers in aqueous solution and its $\mathrm{pH}$ dependence. Biomacromolecules. 2007;8(3):1013-1017.

37. Jeong JH, Byun Y, Park TG. Synthesis and characterization of poly(Llysine)-g-poly(D,L-lactic-co-glycolic acid) biodegradable micelles. J Biomater Sci Polym Ed. 2003;14(1):1-11.

38. Lee SH, Mok H, Lee Y, Park TG. Self-assembled siRNA-PLGA conjugate micelles for gene silencing. J Control Release. 2011;152(1): $152-158$.

39. Kim TI, Lee M, Kim SW. A guanidinylated bioreducible polymer with high nuclear localization ability for gene delivery systems. Biomaterials. 2010;31(7):1798-1804.

40. Jeong JH, Park TG. Novel polymer-DNA hybrid polymeric micelles composed of hydrophobic poly(d,1-lactic-co-glycolic acid) and hydrophilic oligonucleotides. Bioconjug Chem. 2001;12(8):917-923.

41. Mishra D, Kang HC, Bae YH. Reconstitutable charged polymeric (PLGA) $-b$-PEI micelles for gene therapeutics delivery. Biomaterials. 2011;32(15):3845-3854

42. Fu C, Sun X, Liu D, Chen Z, Lu Z, Zhang N. Biodegradable tri-block copolymer poly(lactic acid)-poly(ethylene glycol)-poly(1-lysine) (PLAPEG-PLL) as non-viral vector to enhance gene transfection. Int J Mol Sci. 2011;12(2):1371-1388.

43. Bae Y, Fukushima S, Harada A, Kataoka K. Design of environmentsensitive supramolecular assemblies for intracellular drug delivery: polymeric micelles that are responsive to intracellular $\mathrm{pH}$ change. Angew Chem Int Ed Engl. 2003;42(38):4640-4643.

44. Wang $\mathrm{CH}$, Wang $\mathrm{CH}$, Hsiue GH. Polymeric micelles with a $\mathrm{pH}$-responsive structure as intracellular drug carriers. J Control Release. 2005;108(1):140-149.

45. Reis CP, Neufeld RJ, Ribeiro AJ, Veiga F. Nanoencapsulation I. Methods for preparation of drug-loaded polymeric nanoparticles. Nanomedicine. 2006;2(1):8-21.

46. Yamamoto Y, Nagasaki Y, Kato Y, Sugiyama Y, Kataoka K. Long-circulating poly(ethylene glycol)-poly(lactide) block copolymer micelles with modulated surface charge. $J$ Control Release. 2001;77(1-2):27-38.

47. Sheng Y, Yuan Y, Liu C, Tao X, Shan X, Xu F. In vitro macrophage uptake and in vivo biodistribution of PLA-PEG naoparticles loaded with hemoglobin as blood substitutes: effect of PEG content. J Mater Sci Mater Med. 2009;20(9):1881-1891.

48. Beletsi A, Panagi Z, Avgoustakis K. Biodistribution properties of nanoparticles based on mixtures of PLGA with PLGA-PEG diblock copolymers. Int J Pharm. 2005;298(1):233-241.

49. Verrecchia T, Spenlehauer G, Bazile DV, Murry-Brelier A, Archimbaud Y, Veillard M. Non-stealth (poly(lactic acid/albumin)) and stealth (poly(lactic acid-polyethylene glycol)) nanoparticles as injectable carriers. J Control Release. 1995;36(1-2):49-61. 
50. Suh J, Choy KL, Lai SK, et al. PEGylation of nanoparticles improves their cytoplasmic transport. Int J Nanomedicine. 2007;2(4): 735-741.

51. Pamujulas S, Hazari S, Bolden G, et al. Cellular delivery of PEGylated PLGA nanoparticles. J Pharm Pharmacol. 2012;64(1): 61-67.

52. Li M, Panagi Z, Avgoustakis K, Reineke J. Physiologically based pharmacokinetic modeling of PLGA nanoparticles with varied $\mathrm{mPEG}$ content. Int J Nanomedicine. 2012;7:1345-1356.

53. Amoozgar Z, Yeo Y. Recent advances in stealth coating of nanoparticle drug delivery systems. Wiley Interdiscip Rev Nanomed Nanobiotechnol. 2012;4(2):219-233.

54. Knop K, Hoogenboom R, Fischer D, Schubert US. Poly(ethylene glycol) in drug delivery: pros and cons as well as potential alternatives. Angew Chem Int Ed Engl. 2010;49(36):6288-6308.

55. Gref R, Babak B, Bouillot P, Lukina I, Bodorev M, Dellacherie E. Interfacial and emulsion stabilizing properties of amphiphilic water-soluble poly(ethylene glycol)-poly(lactic acid) copolymers for the fabrication of biocompatible nanoparticles. Colloids Surf A Physicochem Eng Asp. 1998;143(2-3):413-420.

56. Moghimi SM, Szebeni J. Stealth liposomes and long circulating nanoparticles: critical issues in pharmacokinetics, opsonization and protein-binding properties. Prog Lipid Res. 2003;42(6):463-478.

57. Feczkó T, Tóth J, Dósa Gy, Gyenis J. Influence of process conditions on the mean size of PLGA nanoparticles. Chem Eng Process. 2011;50(8): 846-853.

58. Javadzadeh Y, Ahadi F, Davaran S, Mohammadi G, Sabzevari A, Adibkia K. Preparation and physicochemical characterization of naproxen-PLGA nanoparticles. Colloids Surf B Biointerfaces 2010;81(2):498-502.

59. Sanguansri P, Augustin MA, Nanoscale materials development - a food industry perspective. Trends Food Sci Tech. 2006;17(10):547-556.

60. Schubert H, Ax K, Behrend O. Product engineering of dispersed systems. Trends Food Sci Tech. 2003;14(1-2):9-16.

61. Discher DE, Ortiz V, Srinivas G, et al. Emerging application of polymersomes in delivery: From molecular dynamics to shrinkage of tumors. Polym Sci. 2007;32(8-9):838-857.

62. Discher BM, Won YT, Ege DS, et al. Polymersomes: tough vesicles made from diblock copolymers. Science. 1999;284(5417):1143-1146.

63. Discher DE, Eisenberg A. Polymer vesicles. Science. 2002;297(5583): 967-973.

64. Meng F, Hiemstra C, Engbers GHM, Feijen J. Biodegradable polymersomes. Macromolecules. 2003;36(9):3004-3006.

65. Shimada K, Matsuo S, Sadzuka Y, et al. Determination of incorporated amounts of poly(ethylene glycol)-derivatized lipids in liposomes for the physicochemical characterization of stealth liposomes. Int J Pharm. 2000;203(1-2):255-263.

66. Photos PJ, Bacakova L, Discher B, Bates FS, Discher DE. Polymer vesicles in vivo: correlation with PEG molecular weight. J Control Release. 2003;90(3):323-334.

67. Ahmed F, Pakunlu RI, Brannan A, Bates FS, Minko T, Discher DE. Biodegradable polymersomes loaded with both paclitaxel and doxorubicin permeate and shrink tumors, inducing apoptosis in proportion to accumulated drug. J Control Release. 2006;116(2):150-158.

68. Ahmed F, Pakunlu RI, Srinivas G, et al. Shrinkage of a rapidly growing tumor by drug-loaded polymersomes: pH-triggered release through copolymer degradation. Mol Pharm. 2006;3(3):340-350.

69. Rangel-Yagui CO, Pessoa A Jr, Tavares LC. Micellar solubilization of drugs. J Pharm Pharm Sci. 2005;8(2):147-163.

70. Ahmed F, Discher DE. Self-porating polymersomes of PEG-PLA and PEG-PCL: hydrolysis-triggered controlled release vesicles. J Control Release. 2004;96(1):37-53.

71. Chan JM, Zhang LE, Yuet KP, et al. PLGA-lecithin-PEG core-shell nanoparticles for controlled drug delivery. Biomaterials. 2009;30(8): 1627-1634.

72. Zhang L, Zhang L. Lipid-polymer hybrid nanoparticles: synthesis, characterization and applications. Nano Life. 2010;1(1-2):163-173.
73. Thevenot J, Troutier AL, David L, Delair T, Ladaviere C. Steric stabilization of lipid/polymer particle assemblies by poly(ethylene glycol)-lipids. Biomacromolecules. 2007;8(11):3651-3660.

74. Moghimi SM, Hunter AC, Murray JC. Long-circulating and target-specific nanoparticles: theory to practice. Pharmacol Rev. 2001;53(2):283-318.

75. Zhang L, Chan JM, Gu FX, et al. Self-assembled lipid-polymer hybrid nanoparticles: a robust drug delivery platform. ACS Nano. 2008;2(8): 1696-1702.

76. De Miguel I, Imbertie L, Rieumajou V, Major M, Kravtzoff R, Betbeder D. Proofs of the structure of lipid coated nanoparticles (SMBV) used as drug carriers. Pharm Res. 2000;17(7):817-824.

77. Sengupta S, Eavarone D, Capila I, et al. Temporal targeting of tumour cells and neovasculature with a nanoscale delivery system. Nature. 2005;436(7050):568-572.

78. Betancourt T, Brown B, Brannon-Peppas L. Doxorubicin-loaded PLGA nanoparticles by nanoprecipitation: preparation, characterization and in vitro evaluation. Nanomedicine (Lond). 2007;2(2):219-232.

79. Wang Y, Wang R, Lu X, Lu W, Zhang C, Liang W. Pegylated phospholipids-based self-assembly with water-soluble drugs. Pharm Res. 2010;27(2):361-370.

80. Li Y, Taulier N, Rauth AM, Wu XY. Screening of lipid carriers and characterization of drug-polymer-lipid interactions for the rational design of polymer-lipid hybrid nanoparticles (PLN). Pharm Res. 2006;23(8):1877-1887.

81. Ling G, Zhang P, Zhang W, et al. Development of novel self-assembled DS-PLGA hybrid nanoparticles for improving oral bioavailability of vincristine sulfate by P-gp inhibition. J Control Release. 2010;148(2): 241-248.

82. Kreuter J, Gelperina S, Maksimenko O, Khalanskiy A, inventors; LTS Lohmann Therapie-Systeme-AG, assignee. Polylatide nanoparticles. United States patent US 8003128. August 23, 2011.

83. $\mathrm{Mu} \mathrm{L}$, Seow PH.Application of TPGS link polymeric nanoparticulate drug delivery system. Colloids Surf B Biointerfaces. 2006;47(1):90-97.

84. Nafee N, Schneider M, Schaefera UF, Lehra CM. Relevance of the colloidal stability of chitosan/PLGA nanoparticles on their cytotoxicity profile. Int J Pharm. 2009;381(2):130-139.

85. Mansouri S, Lavigne P, Corsi K, Benderdour M, Beaumont E, Fernandes JC. Chitosan-DNA nanoparticles as non-viral vectors in gene delivery: strategies to improve transfection efficacy. Eur J Pharm Biopharm. 2004;57(1):1-8.

86. Nafee N, Taetz S, Schneider M, Schaefer UF, Lehr CM. Chitosancoated PLGA nanoparticles for DNA/RNA delivery: effect of the formulation parameters on complexation and transfection of antisense oligonucleotides. Nanomedicine. 2007;3(3):173-183.

87. Ravi Kumar MN, Bakowsky U, Lehr CM. Preparation and characterization of cationic PLGA nanospheres as DNA carriers. Biomaterials. 2004;25(10):1771-1777.

88. Zhao P, Wang H, Yu M, et al. Paclitaxel loaded folic acid targeted nanoparticles of mixed lipid-shell and polymer-core: in vitro and in vivo evaluation. Eur J Pharm Biopharm. 2012;81(2):248-256.

89. Hu CMJ, Zhang L, Aryal S, Cheung C, Fang RH, Zhang L. Erythrocyte membrane-camouflaged polymeric nanoparticles as a biomimetic delivery platform. Proc Natl Acad Sci U S A. 2011;108(27):10980-10985.

90. Weiss B, Schneider M, Muys L, et al. Coupling of biotin-(poly(ethylene glycol)amine to poly(D,L-lactide-co-glycolide) nanoparticles for versatile surface modification. Bioconjug Chem. 2007;18(4):1087-1094.

91. Pignatello R, Sarpietro MG, Castelli F. Synthesis and biological evaluation of a new polymeric conjugate and nanocarrier with osteotropic properties. J Funct Biomater. 2012;3(1):79-99.

92. Pignatello R, Cenni E, Micieli D, et al. A novel biomaterial for osteotropic drug nanocarriers: synthesis and biocompatibility evaluation of a PLGA-ALE conjugate. Nanomedicine (Lond). 2009;4(2):161-175.

93. Frith JC, Mönkkönen J, Blackburn GM, Russell RG, Rogers MJ. Clodronate and liposome-encapsulated clodronate are metabolized to a toxic ATP analog, adenosine 5'-(beta, gamma-dichloromethylene) triphosphate, by mammalian cells in vitro. J Bone Miner Res. 1997;12(9):1358-1367. 
94. Shmeeda H, Gabizon AA, inventors; Yissum Research Development Company of the Hebrew University of Jerusalem, Ltd, assignee. Targeted liposomes comprising n-containing bisphosphonates and uses of thereof. United States patent application 20120100206. April 26, 2012.

95. Hafeman S, London C, Elmslie R, Dow S. Evaluation of liposomal clodronate for treatment of malignant histiocytosis in dogs. Cancer Immunol Immunother. 2010;59(3):441-452.

96. Guth AM, Hafeman SD, Dow SW. Depletion of phagocytic myeloid cells triggers spontaneous T cell- and NK cell-dependent antitumor activity. OncoImmunology. 2012;1(8):1248-1257.

97. Smith J, Sharma G, inventors. Targeting tumor associated macrophages using bisphosphonate-loaded particles. United States patent application 20110311616. December 22, 2011.

98. Song Q, Yao L, Huang M, et al. Mechanism of transcelluar transport of wheat germ agglutinin-functionalized polymeric nanoparticles in Caco-2-cells. Biomaterials. 2012;33(28):6769-6782.

99. Wang C, Ho PC, Lim LY. Wheat germ agglutinin-conjugated PLGA nanoparticles for enhanced intracellular delivery of paclitaxel to colon cancer cells. Int J Pharm. 2010;400(1-2):201-210.

100. Mo Y, Lim LY. Mechanistic study of the uptake of wheat germ agglutinin-conjugated PLGA nanoparticles by A549 cells. J Pharm Sci. 2004;93(1):20-28.

101. Wen Z, Yan Z, Hu K, et al. Odorranalectin-conjugated nanoparticles: preparation, brain delivery and pharmacodynamic study on Parkinson's disease following intranasal administration. J Control Release. 2011;151(2):131-138.

102. Gao X, Tao W, Lu W, et al. Lectin-conjugated PEG-PLA nanoparticles: preparation and brain delivery after intransal administration. Biomaterials. 2006;27(18):3482-3490.

103. van Kooyk Y. C-type lectins on dendritic cells: key modulators for the induction of immune responses. Biochem Soc Trans. 2008;36(Pt 6):1478-1481.

104. Ghotbi Z, Haddadi A, Hamdy S, Hung RW, Samuel J, Lavasanifar A. Active targeting of dendritic cells with mannan-decorated PLGA nanoparticles. J Drug Target. 2011;19(4):281-292.

105. Tosi G, Vergoni AV, Ruozi B, et al. Sialic acid and glycopeptides conjugated PLGA nanoparticles for central nervous system targeting: in vivo pharmacological evidence and biodistribution. $J$ Control Release. 2010;145(1):49-57.

106. Costantino L, Gandolfi F, Tosi G, Rivasi F, Vandelli MA, Forni F. Peptide-derivatized biodegradable nanoparticles able to cross the blood brain barrier. J Control Release. 2005;108(1):84-96.

107. Michaelis K, Hoffmann MM, Dreis S, et al. Covalent linkage of apolipoprotein $\mathrm{E}$ to albumin nanoparticles strongly enhance drug transport to the brain. J Pharm Exp Ther. 2006;317(3):1246-1253.

108. Alyautdin RN, Petrov VE, Langer K, Berthold A, Kharkevich DA, Kreuter J. Delivery of loperamide across the blood-brain barrier with polysorbate 80 -coated polybutylcyanoacrylate nanoparticles. Pharm Res. 1997;14(3):325-328.

109. Patil Y, Sadhukha T, Ma L, Panyam J. Nanoparticle-mediated simultaneous and targeted delivery of paclitaxel and tariquidar overcomes tumor drug resistance. J Control Release. 2009;136(1):21-29.

110. Patil YB, Swaminathan SK, Sadhukha T, Ma L, Panyam J. The use of nanoparticle-mediated targeted gene silencing and drug delivery to overcome tumor drug resistance. Biomaterials. 2010;31(2):358-365.

111. Ross JF, Chaudhuri PK, Ratnam M. Differential regulation of folate receptor isoforms in normal and malignant tissues. Cancer. 1994; 73(9):2432-2443.

112. Yoo HS, Park TG. Folate receptor targeted biodegradable polymeric doxorubicin micelles. J Control Release. 2004;96(2):273-283.

113. Wang S, Luo Y, Zeng S, et al. Dodeanol-poly(D,L-lactic acid)- $b$ poly(ethylene glycol)-folate (Dol-PLA-PEG-FA) nanoparticles: evaluation of cell cytotoxicity and selecting capability in vitro. Colloids Surf B Biointerfaces. 2013;102(1):130-135.

114. Wang CH, Hsiue GH. Polymer-DNA hybrid nanoparticles based on folate-polyethyleneimine-block-poly(L-lactide). Bioconjug Chem. 2005;16(2):391-396.
115. Esmaeili F, Ghahremani MH, Ostad SB, et al. Folate-receptor-targeted delivery of docetaxel nanoparticles prepared by PLGA-PEG-folate conjugate. J Drug Target. 2008;16(5):415-423.

116. Sunoqrot S, Bae JW, Pearson RM, et al. Temporal control over cellular targeting through hybridization of folate-targeted dendrimers and PEG-PLA nanoparticles. Biomacromolecules. 2012;13(4): 1223-1230.

117. Högemann-Savellano D, Bos E, Blondet C, et al. The transferrin receptor: a potential molecular imaging marker for human cancer. Neoplasia. 2003;5(6):495-506.

118. Shah N, Chaudhari K, Dantuluri P, Murthy RS, Das S. Paclitaxel-loaded PLGA nanoparticles surface modified with transferrin and Pluronic 85, an in vitro cell line and in vivo biodistribution studies on rat model. J Drug Target. 2009;17(7): 533-542.

119. Chang J, Paillard A, Passirani C, et al. Transferrin adsorption onto PLGA nanoparticles governs their interaction with biological systems from blood circulation to brain cancer cells. Pharm Res. 2012;29(8): 1495-1505.

120. Zheng Y, Yu B, Weecharangsan W, et al. Transferrin-conjugated lipid-coated PLGA nanoparticles for targeted delivery of aromatase inhibitor 7 $\alpha$-APTADD to breast cancer cells. Int J Pharm. 2010;390(2): 234-241.

121. Eavarone DA, Yu X, Bellamkonda RV. Targeted drug delivery to C6 glioma by tranferrin-coupled liposomes. J Biomed Mater Res. 2000;51(1):10-14.

122. Yang X, Koh CG, Liu S, et al. Transferrin receptor-targeted lipid nanoparticles for delivery of an antisense oligodeoxyribonucleotide against Bcl-2. Mol Pharm. 2009;6(1):221-230.

123. Hersel U, Dahmen C, Kessler H. RGD modified polymers: biomaterials for stimulated cell adhesion and beyond. Biomaterials. 2003; 24(24):4385-4415.

124. Ding H, Yong KT, Roy I, et al. Bioconjugated PLGA-4-arm-PEG branched polymeric nanoparticles as novel tumor targeting carriers. Nanotechnology. 2011;22(16):165101.

125. Toti US, Guru BR, Grill AE, Panyam J. Interfacial activity assisted surface functionalization: a novel approach to incorporate maleimide functional groups and cRGD peptide on polymeric nanoparticles for targeted drug delivery. Mol Pharm. 2010;7(4):1108-1117.

126. Hrkach JS, Ou J, Lotan N, Langer R. Synthesis of poly(L-lactic acid-co-L-lysine) graft copolymers. Macromolecules. 1995;28(13): 4736-4739.

127. Quirk RA, Chan WC, Davies MC, Tendler SJ, Shakesheff KM. Poly(Llysine)-GRGDS as a biomimetic surface modifier for poly(lactic acid). Biomaterials. 2001;22(8):865-872.

128. Herbig ME, Weller KM, Merkle HP. Reviewing biophysical and cell biological methodologies in cell-penetrating peptide (CPP) research. Crit Rev Ther Drug Carrier Syst. 2007;24(3):203-255.

129. Chugh A, Eudes F, Shim YS. Cell-penetrating peptides: nanocarrier for macromolecule delivery in living cells. IUBMB Life. 2010;62(3): 183-193.

130. Yu DH, Lu Q, Xie J, Fang C, Chen HZ. Peptide-conjugated biodegradable nanoparticles as a carrier to target paclitaxel to tumor neovasculature. Biomaterials. 2010;31(8):2278-2292.

131. Xia H, Gao X, Gu G, et al. Penetratin-functionalized PEG-PLA nanoparticles for brain drug delivery. Int J Pharm. 2012;436(1-2): 840-850.

132. Nam YS, Park JY, Han SH, Chang IS. Intracellular drug delivery using poly(D,L-lactide-co-glycolide) nanoparticles derivatized with a peptide from a transcriptional activator protein of HIV-1. Biotechnol Lett. 2002;24(24):2093-2098.

133. Chen J, Li S, Shen Q. Folic acid and cell-penetrating peptide conjugated PLGA-PEG bifunctional nanoparticles for vincristine sulfate delivery. Eur J Pharm Sci. 2012;47(2):430-443.

134. Sethuraman VA, Lee MC, Bae YH. A biodegradable $\mathrm{pH}$-sensitive micelle system for targeting acidic solid tumors. Pharm Res. 2008;25(3):657-667. 
135. Khondee S, Baoum A, Siahaan TJ, Berkland C. Calcium condensed LABL-TAT complexes effectively target gene delivery to ICAM-1 expressing cells. Mol Pharm. 2011;8(3):788-798.

136. Chittasupho C, Xie SX, Baoum A, Yakovleva T, Siahaan TJ, Berkland CJ. ICAM-1 targeting of doxorubicin-loaded PLGA nanoparticles to lung epithelial cells. Eur J Pharm Sci. 2009;37(2):141-150.

137. Zhang N, Chittasupho C, Duangrat C, Siahaan TJ, Berkland C. PLGA nanoparticle-peptide conjugate effectively targets intercellular celladhesion molecule-1. Bioconjug Chem. 2008;19(1):145-152.

138. Cirstoiu-Hapca A, Bossy-Nobs L, Buchegger F, Gurny R, Delie F. Differential tumor cell targeting of anti-HER2 (Herceptin ${ }^{\circledR}$ ) and anti-CD20 (Mabthera ${ }^{\circledR}$ ) coupled nanoparticles. Int J Pharm. 2007;331(2):190-196.

139. Koopaei MN, Dinarvand R, Amini M, et al. Docetaxel immunonanocarriers as targeted delivery systems for HER2-positive tumor cells: preparation, characterization, and cytotoxicity studies. Int J Nanomedicine. 2011;6:1903-1912.

140. Mitra M, Misra R, Harilal A, Sahoo SK, Krishnakumar S. Enhanced in vitro antiproliferative effects of EpCAM antibody-functionalized paclitaxel-loaded PLGA nanoparticles in retinoblastoma cells. Mol Vis. 2011;17:2724-2737.

141. Muro S, Cui X, Gajewski C, Murciano JC, Myzykantov VR, Koval M. Slow intracellular trafficking of catalse nanoparticles targeted to ICAM-1 protects endothelial cells from oxidative stress. Am JPhysiol Cell Physiol. 2003;285(5):C1339-C1347.

142. Fay F, McLaughlin KM, Small DM, et al. Conatumumab (AMG 655 ) coated nanoparticles for targeted pro-apototic drug delivery. Biomaterials. 2011;32(33):8645-8653.

143. Obemajer N, Kocbek P, Repnik U, et al. Immunonanoparticles - an effective tool to impair harmful proteolysis in invasive breast tumor cells. FEBS J. 2007;274(17):4416-4427.

144. McLoman DP, Barret T, Cummins R, et al. Prognostic significance of TRAIL signaling molecules in stage II and III colorectal cancer. Clin Cancer Res. 2010;16(13):3442-3451.

145. McCarron PA, Marouf WM, Quinn DJ, et al. Antibody targeting of camptothecin-loaded PLGA nanoparticles to tumor cells. Bioconjug Chem. 2008;19(8):1561-1569.

146. Kocbek P, Obermajer N, Cegnar M, Kos J, Kristl J. Targeting cancer cells using PLGA nanoparticles surface modified with monoclonal antibody. J Control Release. 2007;120(1-2):18-26.

147. Nobs L, Buchegger F, Gurny R, Allémann E. Current methods for attaching targeting ligands to liposomes and nanoparticles. J Pharm Sci. 2004;93(8):1980-1992.

148. Shi N, Zhang Y, Zhu C, Boado RJ, Pardrige WM. Brain-specific expression of an exogenous gene after i.v. administration. Proc Natl Acad Sci U S A. 2001;98(22):12754-12759.

149. Olivier JC, Huertas R, Lee HJ, Calon F, Pardridge WM. Synthesis of pegylated immunonanoparticles. Pharm Res. 2002;19(8):137-143.

150. Jaruszewski KM, Ramakrishnan S, Poduslo JF, Kandimalla KK. Chitosan enhances the stability and targeting of immuno-nanovehicles to cerebro-vascular deposits of Alzheimer's disease. Nanomedicine. 2012;8(2):250-260.

151. Kempf M, Mandal B, Jilek S, et al. Improved simulation of human dendritic cells by receptor engagement with surface-modified microparticles. J Drug Target. 2003;11(1):11-18.
152. Wei H, Wang S, Zhang D, et al. Targeted delivery of tumor antigens to activated dendritic cells via CD11c molecules induces potent antitumor immunity in mice. Clin Cancer Res. 2009;15(14):4612-4621.

153. Barbas AS, Mi J, Clary BM, White RR. Aptamer applications for targeted cancer therapy. Future Oncol. 2010;6(7):1117-1126.

154. Ng E, Shima D, Calias P, Cunningham E Jr, Guyer D, Adamis A. Pegaptanib, a targeted anti-VEGF aptamer for ocular vascular disease. Nat Rev Drug Discov. 2006;5(2):123-132.

155. Hovanessian AG, Soundaramourty C, El KD, Nondier I, Svab J, Krust B. Surface expressed nucleolin is constantly induced in tumor cells to mediate calcium dependent ligand internalization. PLoS One. 2010;5(12):e15787.

156. Hwang DW, Ko HY, Lee JH, et al. A nucleolin-targeted multimodal nanoparticle imaging probe for tracking cancer cells using an aptamer. J Nucl Med. 2010;51(1):98-105.

157. Reyes-Reyes EM, Teng Y, Bates PL. A new paradigm for aptamer therapeutic AS1411 action: uptake by macropinocytosis and its stimulation by a nucleolin-dependent mechanism. Cancer Res. 2010;70(21):8617-8629.

158. Guo J, Gao X, Su L, et al. Aptamer-functionalized PEG-PLGA nanoparticles for enhanced anti-glioma drug delivery. Biomaterials. 2011;32(31):10-20.

159. Aravind A, Jeyamothan P, Nair R, et al. AS1411 aptamer tagged PLGA-lecithin-PEG nanoparticles for tumor cell targeting and drug delivery. Biotechnol Bioeng. 2012;109(11):2920-2931.

160. Chan JM, Valencia PM, Zhang L, Langer R, Farokhzad OC. Polymeric nanoparticles for drug delivery. Methods Mol Biol. 2010;624:163-175.

161. Gu F, Langer R, Farokhzad OC. Formulation/preparation of functionalized nanoparticles for in vivo targeted drug delivery. Methods Mol Biol. 2009;544:589-598.

162. Farokhzad OC, Jon S, Khademhosseini A, Tran TNT, LaVan DA, Langer R. Nanoparticle-aptamer bioconjugates: a new approach for targeting prostate cancer cells. Cancer Res. 2004;64(21):7668-7672.

163. Wang L, Neoh K, Kang E, Shuter B, Wang S. Biodegradable magnetic-fluorescent magnetite/poly(dl-lactic acid-co- $\alpha, \beta$-malic acid) composite nanoparticles for stem cell labeling. Biomaterials. 2010;31(13):3502-3511.

164. Lim YT, Noh Y, Han JH, Cai Q, Yoon K, Chung BH. Biocompatible polymer-nanoparticle-based bimodal imaging contrast agents for the labeling and tracking of dendritic cells. Small. 2008;4(10): 1640-1645.

165. Chen Z, Yu D, Liu C, et al. Gadolinium-conjugated PLA-PEG nanoparticles as liver targeted molecular MRI contrast agent. J Drug Target. 2011;19(8):657-665.

166. Doiron AL, Homan KA, Emelianov S, Brannon-Peppas L. Poly(lacticco-glycolic) acid as a carrier for imaging contrast agents. Pharm Res. 2009;26(3):674-682.

167. Lee SJ, Jeong JR, Shin SC, et al. Intracellular translocation of superparamagnetic iron oxide nanoparticles encapsulated with peptide-conjugated poly (D,L lactide-co-glycolide). J Appl Phys. 2005;97(10):Q913-1-Q913-3.

168. Yang J, Lee CH, Park J, et al. Antibody conjugated magnetic PLGA nanoparticles for diagnosis and treatment of breast cancer. J Mater Chem. 2007;17:2695-2699.

International Journal of Nanomedicine

\section{Publish your work in this journal}

The International Journal of Nanomedicine is an international, peerreviewed journal focusing on the application of nanotechnology in diagnostics, therapeutics, and drug delivery systems throughout the biomedical field. This journal is indexed on PubMed Central,

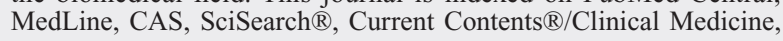

Journal Citation Reports/Science Edition, EMBase, Scopus and the Elsevier Bibliographic databases. The manuscript management system is completely online and includes a very quick and fair peer-review system, which is all easy to use. Visit http://www.dovepress.com/ testimonials.php to read real quotes from published authors. 